A Linchpin Approach to Unsaturated

Fatty Acids: 11,12-

Epoxyeicosatrienoic Acid and

11S,12S-Dihydroxyeicosatrienoic Acid

\section{Ethyl Esters}

\section{(Supporting Information)}

Douglass F. Taber,* and Zhe Zhang

Department of Chemistry and

Biochemistry, University of Delaware,

Newark, Delaware 19716

taberdf@udel.edu

\section{Table of Contents}

S-2 General Experimental Procedures

S-7 ${ }^{1} \mathrm{H}$ NMR for 11

S-8 ${ }^{13} \mathrm{C}$ NMR for 11

S-9 ${ }^{~} \mathrm{H}$ NMR for 12

$\mathrm{S}-10{ }^{13} \mathrm{C}$ NMR for 12

S-11 ${ }^{1} \mathrm{H}$ NMR for 13

S- $12{ }^{13} \mathrm{C}$ NMR for 13

S-13 ${ }^{1} \mathrm{H}$ NMR for 14
S-14 ${ }^{13} \mathrm{C}$ NMR for 14

S- $15{ }^{1}$ H NMR for 8

$\mathrm{S}-16{ }^{13} \mathrm{C}$ NMR for 8

S- $17{ }^{1} \mathrm{H}$ NMR for 15

S-18 ${ }^{13} \mathrm{C}$ NMR for 15

S-19 ${ }^{1} \mathrm{H}$ NMR for 16

$\mathrm{S}-20{ }^{13} \mathrm{C}$ NMR for 16

S-21 ${ }^{\mathrm{I}} \mathrm{H}$ NMR for 17

S-22 ${ }^{13} \mathrm{C}$ NMR for 17

S-23 ${ }^{1} \mathrm{H}$ NMR for 18

S- $24{ }^{13} \mathrm{C}$ NMR for 18

S-25 ${ }^{1}$ H NMR for 9

S-26 ${ }^{13} \mathrm{C}$ NMR for 9

S-27 ${ }^{1} \mathrm{H}$ NMR for Methyl Ester of 9

S-28 ${ }^{13} \mathrm{C}$ NMR for Methyl Ester of 9 


\section{General Experimental Procedures}

${ }^{1} \mathrm{H}$ NMR and ${ }^{13} \mathrm{C}$ NMR spectra were obtained as solutions in deuteriochloroform $\left(\mathrm{CDCl}_{3}\right)$ ar $400 \mathrm{MHz}$ and $100 \mathrm{MHz}$, respectively, with tetramethylsilane $=0.00$ as an internal standard. ${ }^{13} \mathrm{C}$ multiplicities were determined with the aid of a JVERT pulse sequence, differentiating the signals for methyl and methine carbons as " $\mathrm{d}$ " from methylene and quaternary carbons as " $u$ ". The infrared (IR) spectra were determined as neat oils. Optical rotations were determined as solutions in dichloromethane unless otherwise noted. $R_{\mathrm{f}}$ values indicated refer to thin layer chromatography (TLC) on $2.5 \times 10 \mathrm{~cm}, 250$ $\mu \mathrm{m}$ analytical plates coated with silica gel GF, unless otherwise noted, and developed in the solvent system indicated. The solvent mixtures used are volume/volume mixtures. All glassware was oven dried before use. Tetrahydrofuran (THF) was distilled from sodium metal/benzophenone ketyl under dry nitrogen. Dichloromethane $\left(\mathrm{CH}_{2} \mathrm{Cl}_{2}\right)$ was distilled from calcium hydride under dry nitrogen. MTBE is methyl t-butyl ether. PE is petroleum ether. All reactions were performed under a nitrogen atmosphere unless otherwise specified. 
trans-Enyne alcohol (15): The same procedure was applied as the preparation of 11 except that the trans-1,4-dichloro-2-butene 5 was used. trans-Enyne alcohol 15 was prepared as a colorless oil (47\% yield from 1-heptyne), TLC $R_{\mathrm{f}}(\mathrm{MTBE} /$ petroleum ether = $1: 4)=0.45 ;{ }^{1} \mathrm{H}$ NMR $\delta 0.86(\mathrm{t}, \mathrm{J}=7.1 \mathrm{~Hz}, 3 \mathrm{H}), 1.30-1.33(\mathrm{~m}, 4 \mathrm{H}), 1.44-1.47(\mathrm{~m}, 2 \mathrm{H})$, $1.95(\mathrm{t}, \mathrm{J}=6.4 \mathrm{~Hz}, 1 \mathrm{H}), 2.13(\mathrm{t}, J=7.2 \mathrm{~Hz}, 2 \mathrm{H}), 2.88$ (br s, 2H), 2.94 (br s, 2H), 4.25 (d, $\mathrm{J}=6.4 \mathrm{~Hz}, 2 \mathrm{H}), 5.48-5.52(\mathrm{~m}, 2 \mathrm{H}) ;{ }^{13} \mathrm{C}$ NMR $\delta \mathrm{u} 18.7,21.6,21.7,22.1,28.6,31.0,51.2$, $76.7,80.2,82.6,83.3 ; \mathrm{d} 13.9,125.0,126.9 ; \mathrm{IR}\left(\mathrm{cm}^{-1}\right) ; 3152,2956,2930 ; \mathrm{MS} \mathrm{m} / z(\%)$ : 203(M-H, 17), 91(100); HRMS Calcd for $\mathrm{C}_{14} \mathrm{H}_{21} \mathrm{O}(\mathrm{M}+\mathrm{H})$ 205.1592, Obsd 205.1594.

trans-Triyne (16): The same procedure was applied as the preparation of 12 . transTriyne 16 was prepared as a colorless oil ( $40 \%$ yield from 15), TLC $R_{\mathrm{f}}(\mathrm{MTBE} /$ petroleum ether $=1: 10)=0.35 ;{ }^{1} \mathrm{H}$ NMR $\delta 0.86(\mathrm{t}, \mathrm{J}=7.1 \mathrm{~Hz}, 3 \mathrm{H}), 1.30-1.33(\mathrm{~m}, 4 \mathrm{H}), 1.44-1.49$ $(\mathrm{m}, 2 \mathrm{H}), 1.79-1.86(\mathrm{~m}, 2 \mathrm{H}), 2.13-2.16(\mathrm{~m}, 2 \mathrm{H}), 2.31-2.33(\mathrm{~m}, 2 \mathrm{H}), 2.47(\mathrm{t}, \mathrm{J}=7.2 \mathrm{~Hz}$, 2H), 2.90 (br s, 4H), 3.13 (br s, $2 \mathrm{H}), 5.66$ (br s, $2 \mathrm{H}$ ); ${ }^{13} \mathrm{C}$ NMR $\delta$ u 9.7, 16.1, 17.8, 18.7, $21.6,21.7,22.2,24.6,28.7,31.1,76.0,76.5,76.8,77.6,77.8,82.6,119.2 ; \mathrm{d} 14.0,125.4$

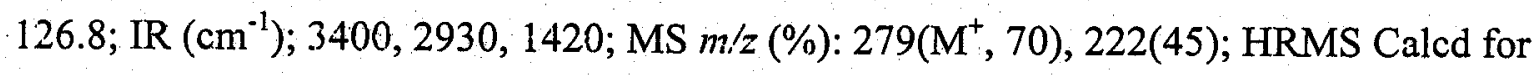
$\mathrm{C}_{20} \mathrm{H}_{25} \mathrm{NNa}(\mathrm{M}+\mathrm{Na}) 302.1885$, Obsd 302.1871.

trans-Lster (17): The same procedure was applied as the preparation of 13. The transester 17 was prepared as a colorless oil (71\% yield from 16): TLC $R_{\mathrm{f}}(\mathrm{MTBE} /$ petroleum 
ether $=1: 30)=0.45 ;{ }^{1} \mathrm{H}$ NMR $\delta 0.87(\mathrm{t}, \mathrm{J}=6.9 \mathrm{~Hz}, 3 \mathrm{H}), 1.23(\mathrm{t}, \mathrm{J}=7.1 \mathrm{~Hz}, 3 \mathrm{H}), 1.30-$ $1.36(\mathrm{~m}, 4 \mathrm{H}), 1.43-1.49(\mathrm{~m}, 2 \mathrm{H}), 1.75-1.82(\mathrm{~m}, 2 \mathrm{H}), 2.12-2.17(\mathrm{~m}, 2 \mathrm{H}), 2.19-2.23(\mathrm{~m}$, $2 \mathrm{H}$ ), 2.39 (t, J = 7.5 Hz, 2H), 2.89 (br s, 4H), 3.12-3.13 (m, 2H), 4.10 (q, J = 7.5 Hz, 2H), 5.65 (br s, 2H); ${ }^{13} \mathrm{C}$ NMR $\delta$ u 9.7, 18.2, 18.7, 21.6, 21.7, 22.2, 23.9, 28.7, 31.1, 33.1, 60.3, $75.2,76.3,76.8,77.6,79.3,82.6,173.2 ; \mathrm{d} 14.0,14.2,125.5,126.7$; IR $\left(\mathrm{cm}^{-1}\right) ; 2932$, 1732, 1158; MS m/z (\%): 297( $\mathrm{M}^{+}$-Et, 10), 155(100); HRMS Calcd for $\mathrm{C}_{22} \mathrm{H}_{30} \mathrm{O}_{2} \mathrm{Na}$ $(\mathrm{M}+\mathrm{Na}) 349.2144$, Obsd 349.2149.

Diol (18): A suspension of AD-mix- $\alpha(1.47 \mathrm{~g})$ in $t-\mathrm{BuOH}-\mathrm{H}_{2} \mathrm{O}(1: 1, \mathrm{v} / \mathrm{v}, 8 \mathrm{~mL})$ was stirred at rt until both phases were clear. The mixture was cooled to $0^{\circ} \mathrm{C}$ and methanesulforamide $(70 \mathrm{mg}, 0.74 \mathrm{mmol})$ was added, followed by the trans-ester 17 (212 $\mathrm{mg}, 0.65 \mathrm{mmol})$ in MTBE $(0.5 \mathrm{~nL})$ dropwise over $5 \mathrm{~min}$. The resulting mixture was stirred at $0^{\circ} \mathrm{C}$ for $4.9 \mathrm{~h}$. Solid $\mathrm{NaISSO}_{3}(1.52 \mathrm{~g})$ was carefully added to the reaction mixture. The mixture was stirred at $\mathrm{rt}$ for $1 \mathrm{~h}$, then partitioned between EtOAc and brine. The combined organic extracts were dried $\left(\mathrm{Na}_{2} \mathrm{SO}_{4}\right)$ and concentrated. The residue was chromategraphed to give diol 18 as a colorless oil (122 mg, $0.34 \mathrm{mmol}, 52 \%$ yield). TLC $R_{f}(\mathrm{MTBE} /$ petroleum ether $=1: 1)=0.20 ;{ }^{1} \mathrm{H}$ NMR $\delta 0.87(\mathrm{t}, \mathrm{J}=7.2 \mathrm{~Hz}, 3 \mathrm{H}), 1.23(\mathrm{t}, \mathrm{J}=$ $7.1 \mathrm{~Hz}, 3 \mathrm{H}) ; 1.39-1.33(\mathrm{~m}, 4 \mathrm{H}), 1.42-1.47(\mathrm{~m}, 2 \mathrm{H}), 1.74-1.82(\mathrm{~m}, 2 \mathrm{H}), 2.10-2.15(\mathrm{~m}, 2 \mathrm{H})$, 2.18-2.23 (m, 2H); $2.39(\mathrm{t}, \mathrm{J}=7.5 \mathrm{~Hz}, 2 \mathrm{H}), 2.43-2.53(\mathrm{~m}, 4 \mathrm{H}), 3.09-3.11(\mathrm{~m}, 2 \mathrm{H}), 3.70-$ $3.77(\mathrm{~m}, 2 \mathrm{H}), 4.10(\mathrm{q}, \mathrm{J}=7.5 \mathrm{~Hz}, 2 \mathrm{I}) ;{ }^{13} \mathrm{C}$ iNMR $\delta$ u 9.8, 18.1, 18.7, 22.2, 23.8, 24.2, 
$24.3,28.6,31.1,33.1,60.4,74.9,75.4,76.3,77.2,79.5,83.5,173.3 ; \mathrm{d} 14.0,14.2,71.1$, 71.2; IR $\left(\mathrm{cm}^{-1}\right) ; 3224,2932,1736$; MS m/z (\%): 361( $\left.\mathrm{M}^{+}+\mathrm{H}, 100\right), 343(30)$; HRMS Calcd

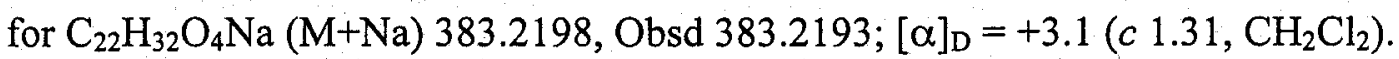

Triene (9): To a solution of $\mathrm{Ni}(\mathrm{OAc})_{2} \bullet 4 \mathrm{H}_{2} \mathrm{O}(34 \mathrm{mg}, 0.14 \mathrm{mmol})$ in ethanol $(0.5 \mathrm{~mL})$ was add a solution of $\mathrm{NaBH}_{4}(1 \mathrm{M}$ solution in ethanol, $0.12 \mathrm{~mL}, 0.12 \mathrm{mmol})$. The black mixture was evacuated and backfilled with $\mathrm{H}_{2}$ three times. Ethylenediamine $(9 \mathrm{mg}, 0.15$ $\mathrm{mmol}, 0.01 \mathrm{~mL})$ was added, followed by a solution of diol $18(100 \mathrm{mg}, 0.28 \mathrm{mmol})$ in ethanol $(0.5 \mathrm{~mL})$. The flask was evacuated and backfilled with $\mathrm{H}_{2}$ three times. The reaction mixture was stirred at $\mathrm{rt}$ for $10 \mathrm{~h}$ under $\mathrm{H}_{2}$. The black suspension was filtered through a short column packed with flash silica gel. The column was eluted with MTBE $(50 \mathrm{~mL})$. The solvent was removed to give the triene 9 as a colorless oil $(82 \mathrm{mg}, 0.22$ mmol, $80 \%$ yield). TLC $R_{\mathrm{f}}(\mathrm{MTBE} /$ petroleum ether $=3: 1)=0.15 ;{ }^{1} \mathrm{H} \mathrm{NMR} \delta 0.86(\mathrm{t}, \mathrm{J}=$ $7.0 \mathrm{~Hz}, 3 \mathrm{H}), 1.23(\mathrm{t}, \mathrm{J}=7.1 \mathrm{~Hz}, 3 \mathrm{H}), 1.25-1.37(\mathrm{~m}, 6 \mathrm{H}), 1.62-1.70(\mathrm{~m}, 2 \mathrm{H}), 2.00-2.11(\mathrm{~m}$, $4 \mathrm{H}), 2.26-2.32(\mathrm{~m}, 6 \mathrm{H}), 2.78-2.81(\mathrm{~m}, 2 \mathrm{H}), 3.49-3.52(\mathrm{~m}, 2 \mathrm{H}), 4.10(\mathrm{q}, \mathrm{J}=7.1 \mathrm{~Hz}, 2 \mathrm{H})$, 5.34-5.53 (m, 6H); ${ }^{13} \mathrm{C}$ NMR $\delta$ u $22.5,24.8,25.8,26.6,27.4,29.3,31.5,31.8,31.9,33.7$, $60.3,173.8 ; \mathrm{d} 14.0,14.2,73.1,73.2,124.6,125.3,128.5,129.1,131.1,133.6 ; \mathrm{IR}\left(\mathrm{cm}^{-1}\right)$; 3224, 2926, 1736; MS $m / z(\%): 367\left(\mathrm{M}^{+}+\mathrm{H}, 100\right), 242(20)$; HRMS Calcd for $\mathrm{C}_{22} \mathrm{H}_{38} \mathrm{O}_{4} \mathrm{Na}$ $(\mathrm{M}+\mathrm{Na}) 389.2668$, Obsd 389.2666; $[\alpha]_{\mathrm{D}}=-16.6\left(c 0.51, \mathrm{CH}_{2} \mathrm{Cl}_{2}\right)$. 
Methyl ester: To a solution of triene $9(50 \mathrm{mg}, 0.14 \mathrm{mmol})$ in $\mathrm{MeOH}(0.5 \mathrm{~mL})$ was added $\mathrm{K}_{2} \mathrm{CO}_{3}(19 \mathrm{mg}, 0.14 \mathrm{mmol})$ and the mixture was stirred at $\mathrm{rt}$ for $1.5 \mathrm{~h}$, then partitioned between $\mathrm{CH}_{2} \mathrm{Cl}_{2}$ and brine. The combined organic extracts were dried $\left(\mathrm{Na}_{2} \mathrm{SO}_{4}\right)$ and concentrated. The residue was chromatographed to give the methyl ester as a colorless oil ( $40 \mathrm{mg}, 0.11 \mathrm{mmol}, 83 \%$ yield). TLC $\mathrm{R}_{\mathrm{f}}(\mathrm{MTBE} /$ petroleum ether $=3: 1$ ) $=0.15 ;{ }^{1} \mathrm{H}$ NMR $\delta 0.86(\mathrm{t}, \mathrm{J}=7.0 \mathrm{~Hz}, 3 \mathrm{H}), 1.26-1.35(\mathrm{~m}, 6 \mathrm{H}), 1.62-1.70(\mathrm{~m}, 2 \mathrm{H}), 2.02-$ $2.07(\mathrm{~m}, 4 \mathrm{H}), 2.16-2.32(\mathrm{~m}, 6 \mathrm{H}), 2.78-2.80(\mathrm{~m}, 2 \mathrm{H}), 3.48-3.51(\mathrm{~m}, 2 \mathrm{H}), 3.64(\mathrm{~s}, 3 \mathrm{H})$, 5.35-5.53 (m, 6H); ${ }^{13} \mathrm{C}$ NMR $\delta$ u 22.5, 24.7, 25.7, 26.6, 27.2, 29.3, 31.5, 31.8, 31.9, 33.4, 174.0; d 14.0, 51.5, 73.1, 73.2, 124.6, 125.3, 128.6, 129.0, 131.1, 133.6; IR $\left(\mathrm{cm}^{-1}\right) ; 3216$, 2926, 1740; MS m/z (\%): 353(M+H, 30), 225(50); HRMS Calcd for $\mathrm{C}_{21} \mathrm{H}_{36} \mathrm{O}_{4} \mathrm{Na}$ $(\mathrm{M}+\mathrm{Na})$ 375.2511, Obsd 375.2515; $[\alpha]_{\mathrm{D}}=-6.1\left(\mathrm{c} 1.05, \mathrm{CHCl}_{3}\right)$, lit: $[\alpha]_{\mathrm{D}}=-6.3(\mathrm{c} 0.97$, $\left.\mathrm{CHCl}_{3}\right)$. 

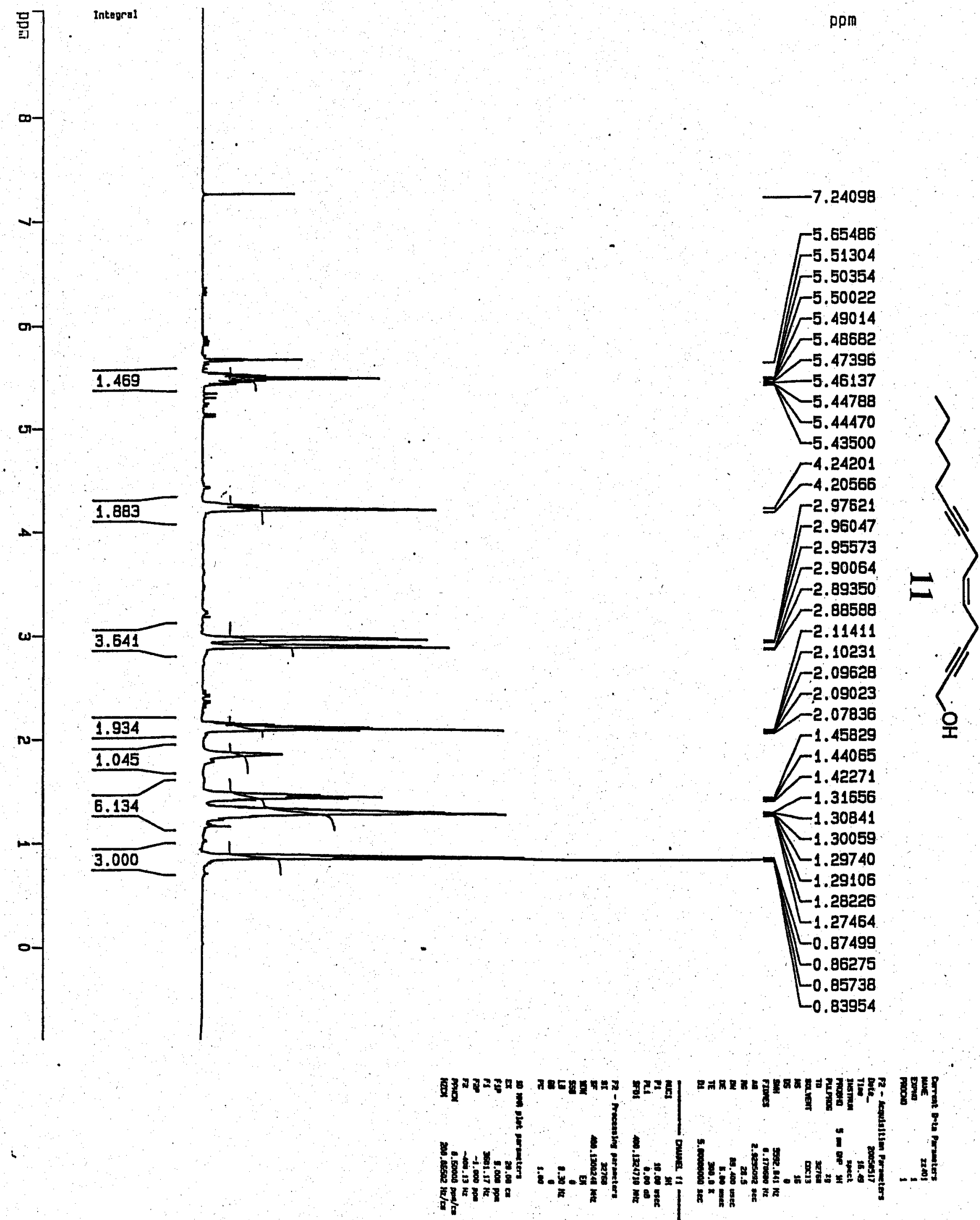


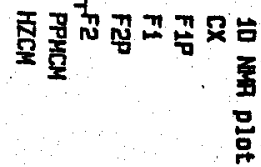

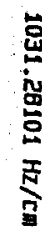

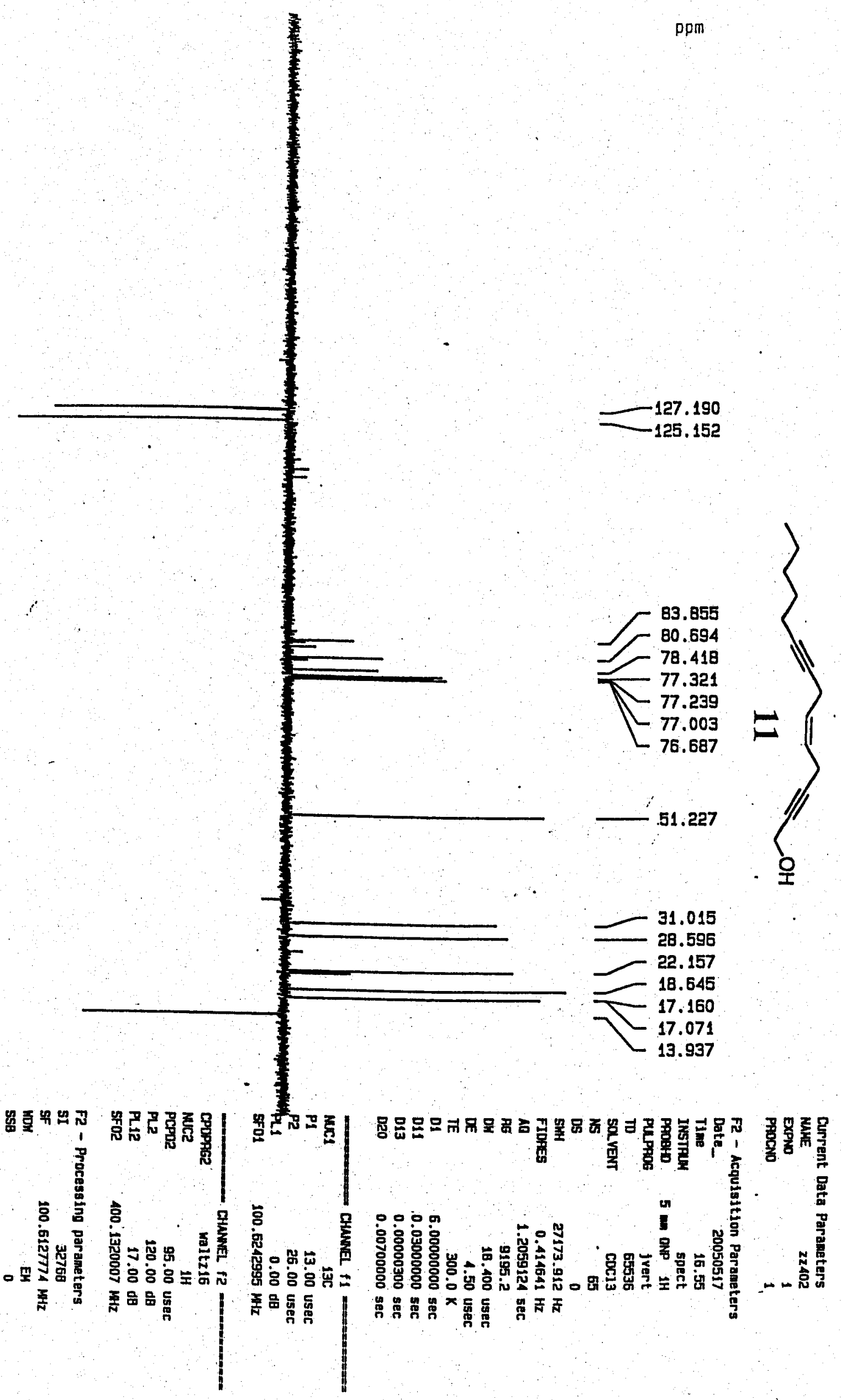



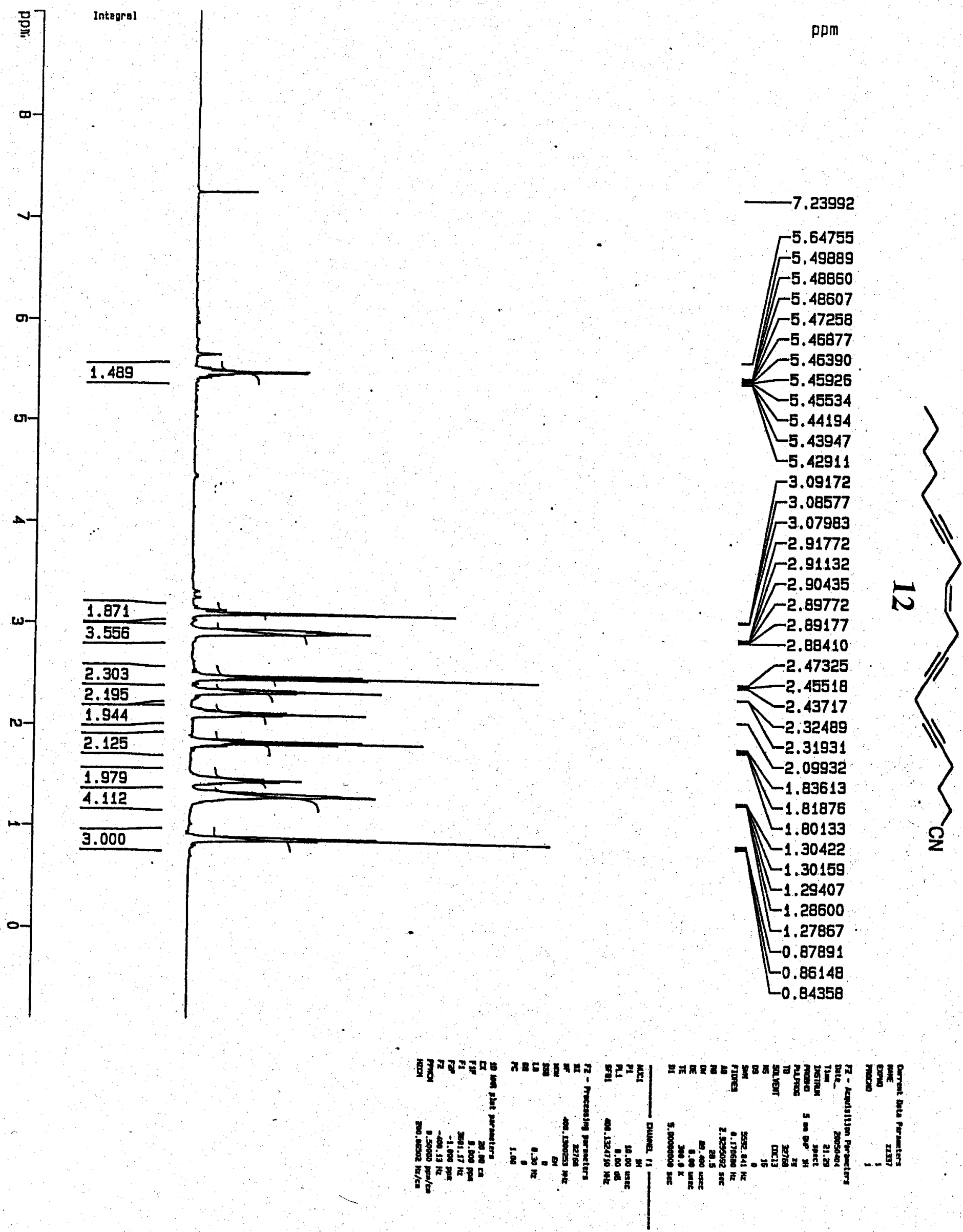


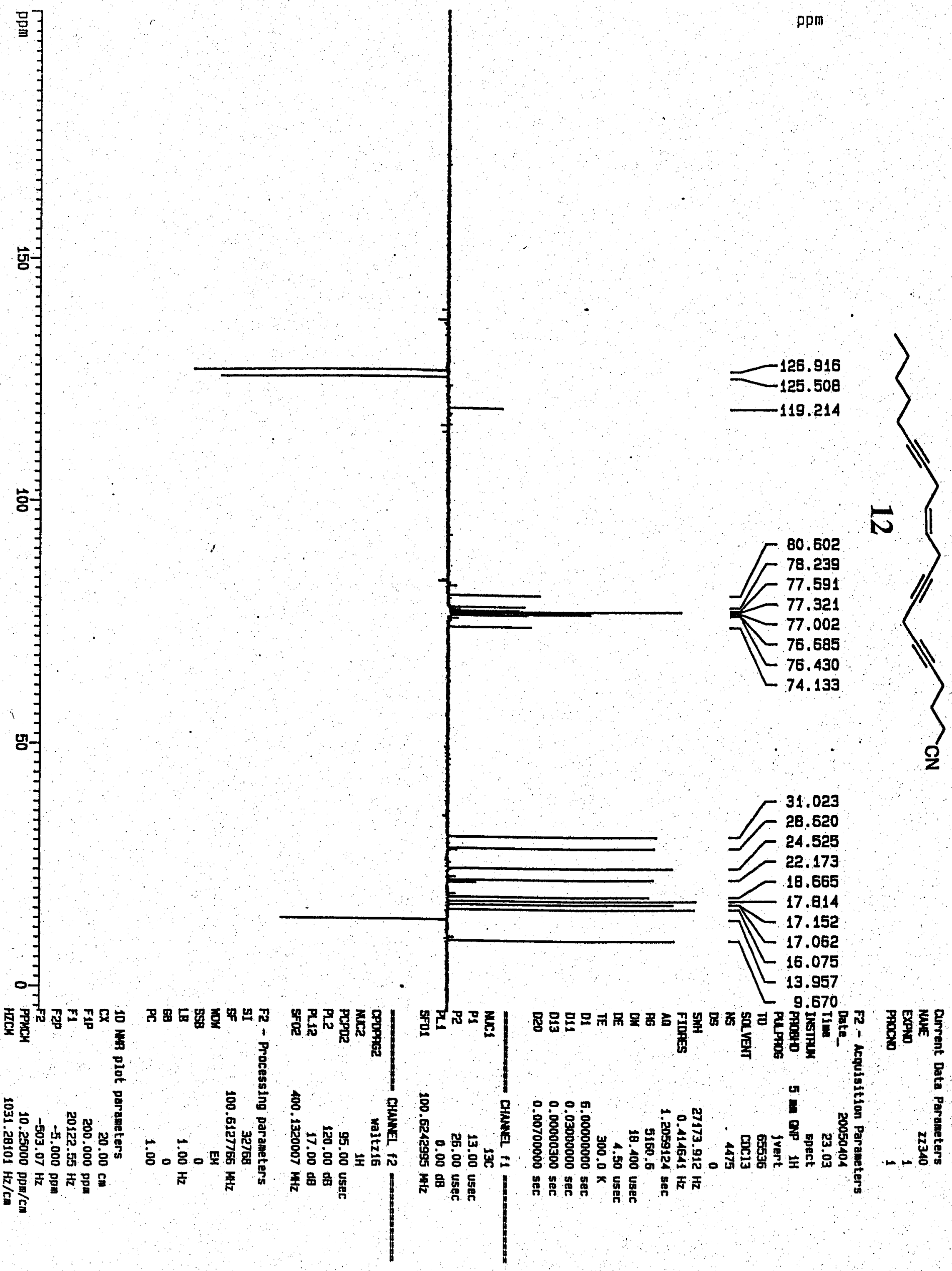



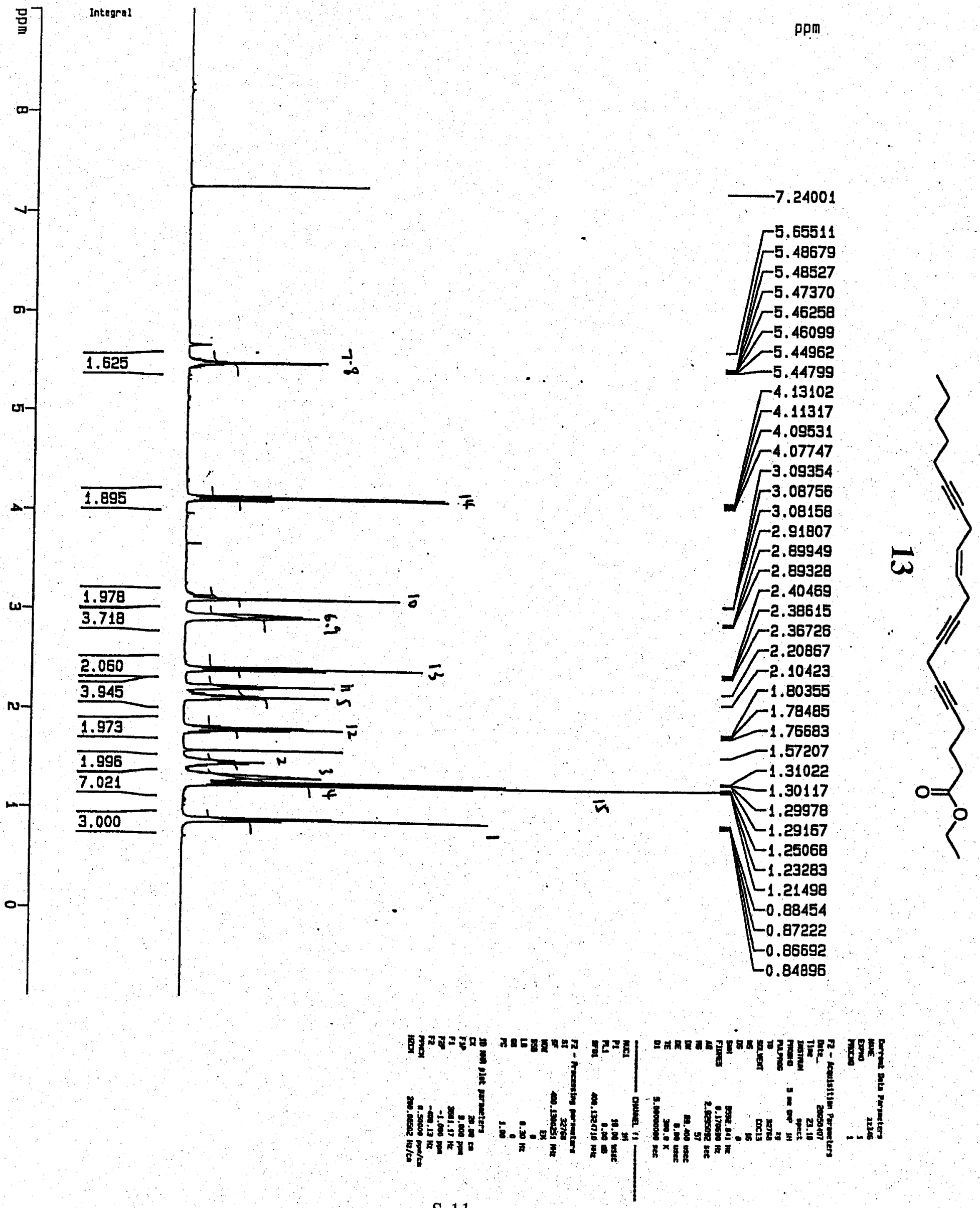


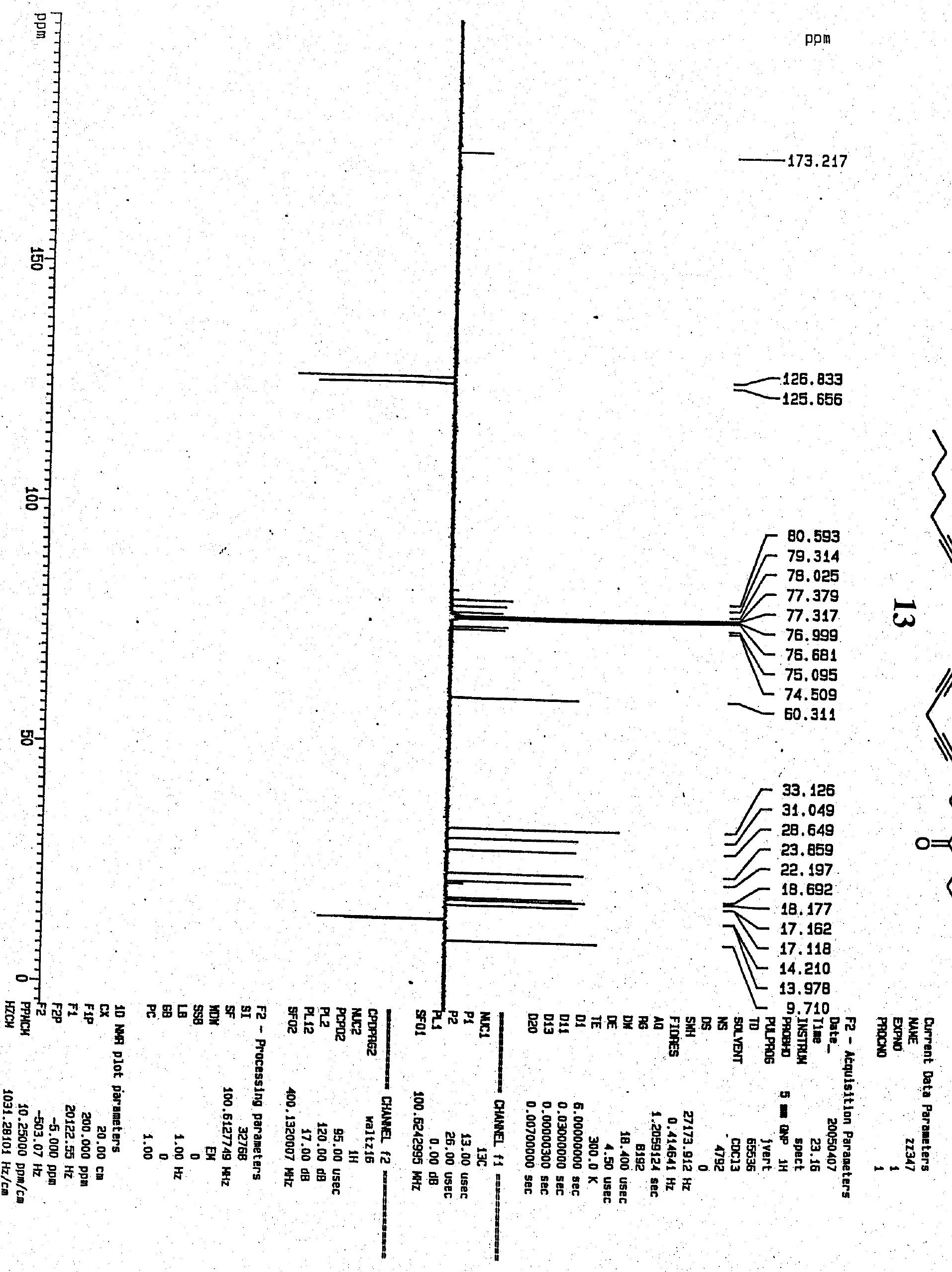



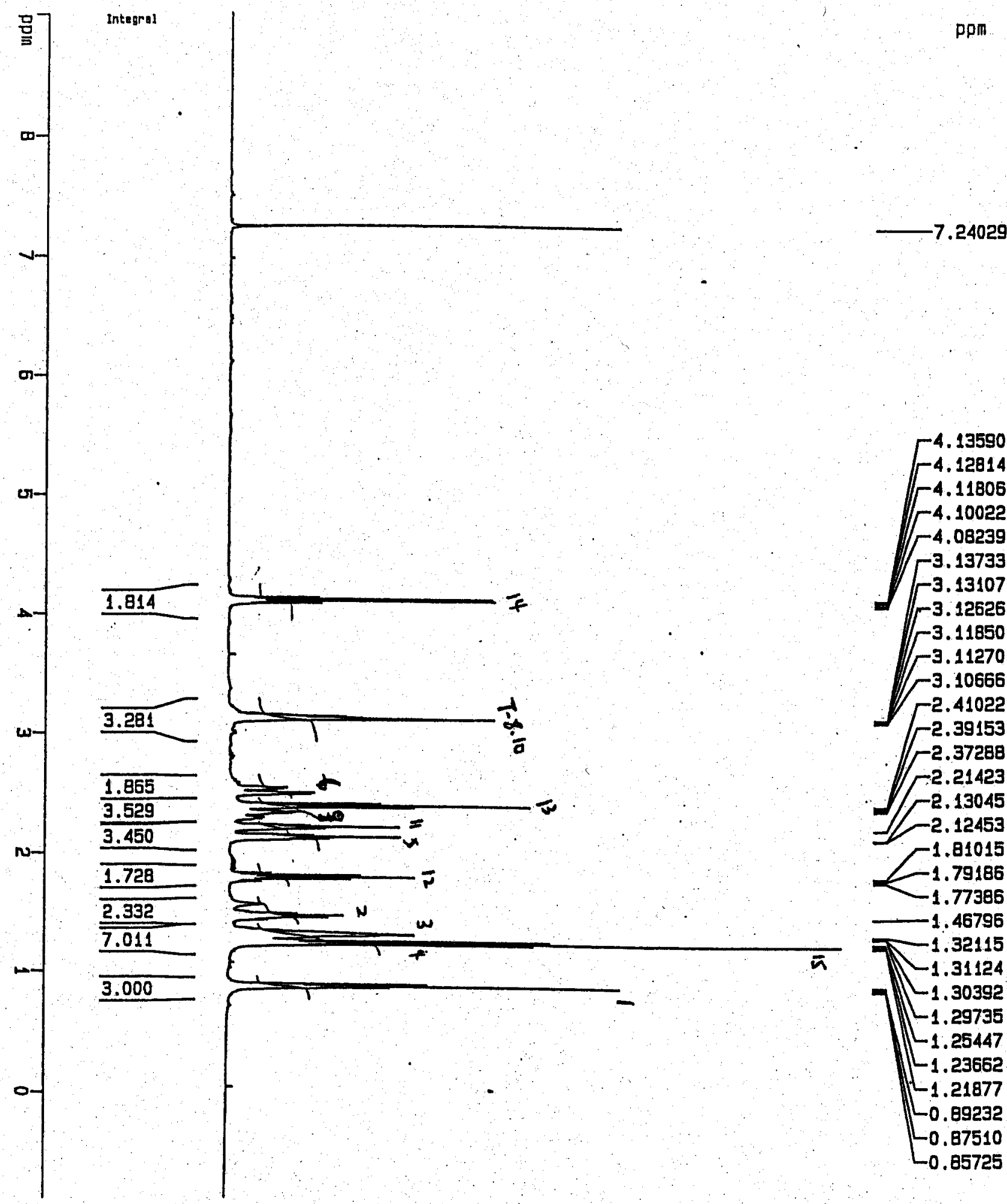

$-4.12814$

$-4.11806$

4.10022

$-4.08239$

$-3.13733$

$-3.13107$

$-3.12626$

$-3.11850$

$r^{3.11270}$

$-3.10666$

2.41022

$-2.39153$

$-2.37288$

-2.21423

$-2.13045$

$-2.12453$

$-1.81015$

$-1.79186$

$-1.77386$

1.46796

$-1.32115$

$7-1.31124$

$-1.30392$

$-1.29735$

$-1.25447$

$-1.23662$

$-1.21877$

$L_{0.89232}$

$-0.87510$

$A$
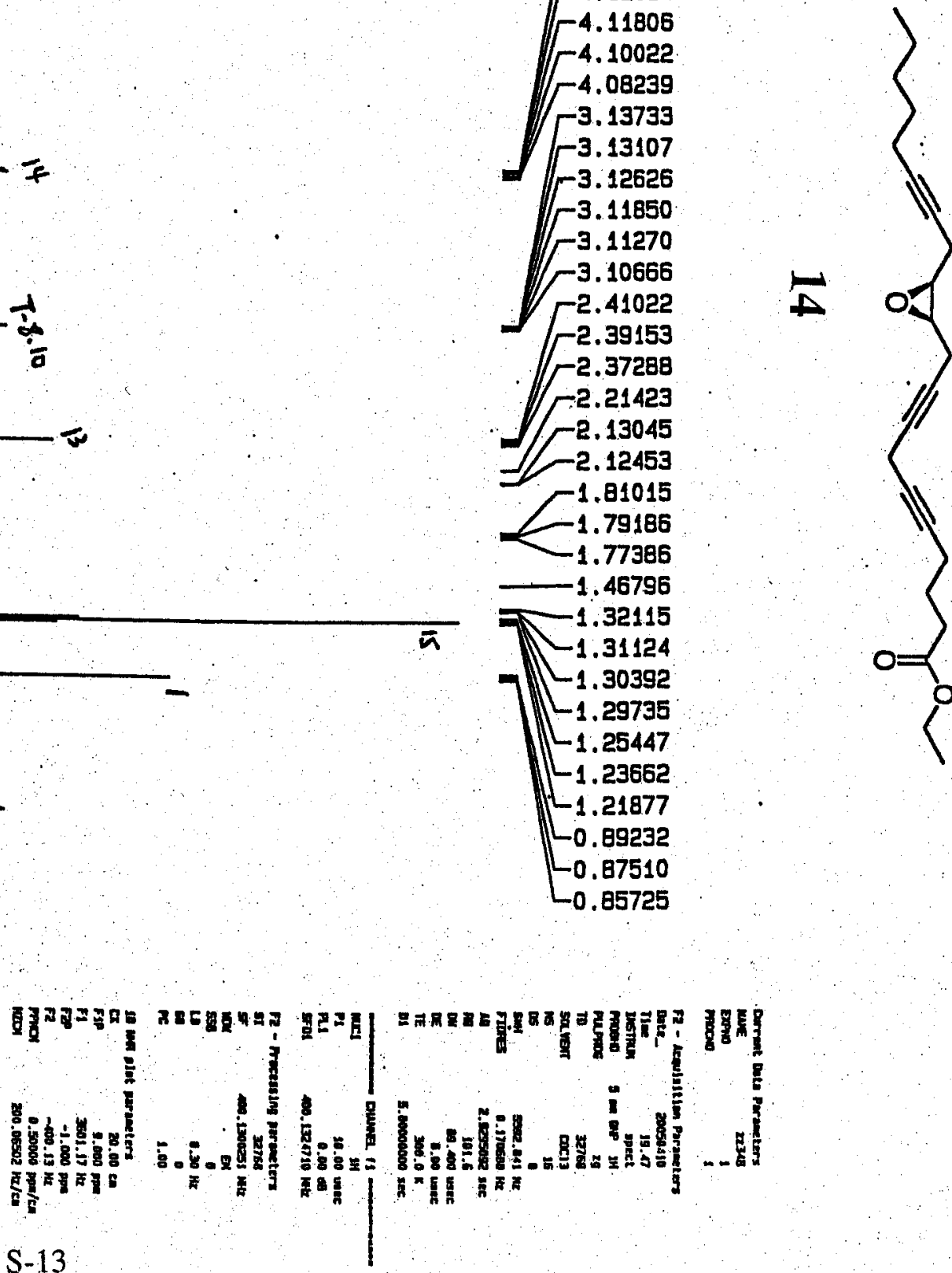


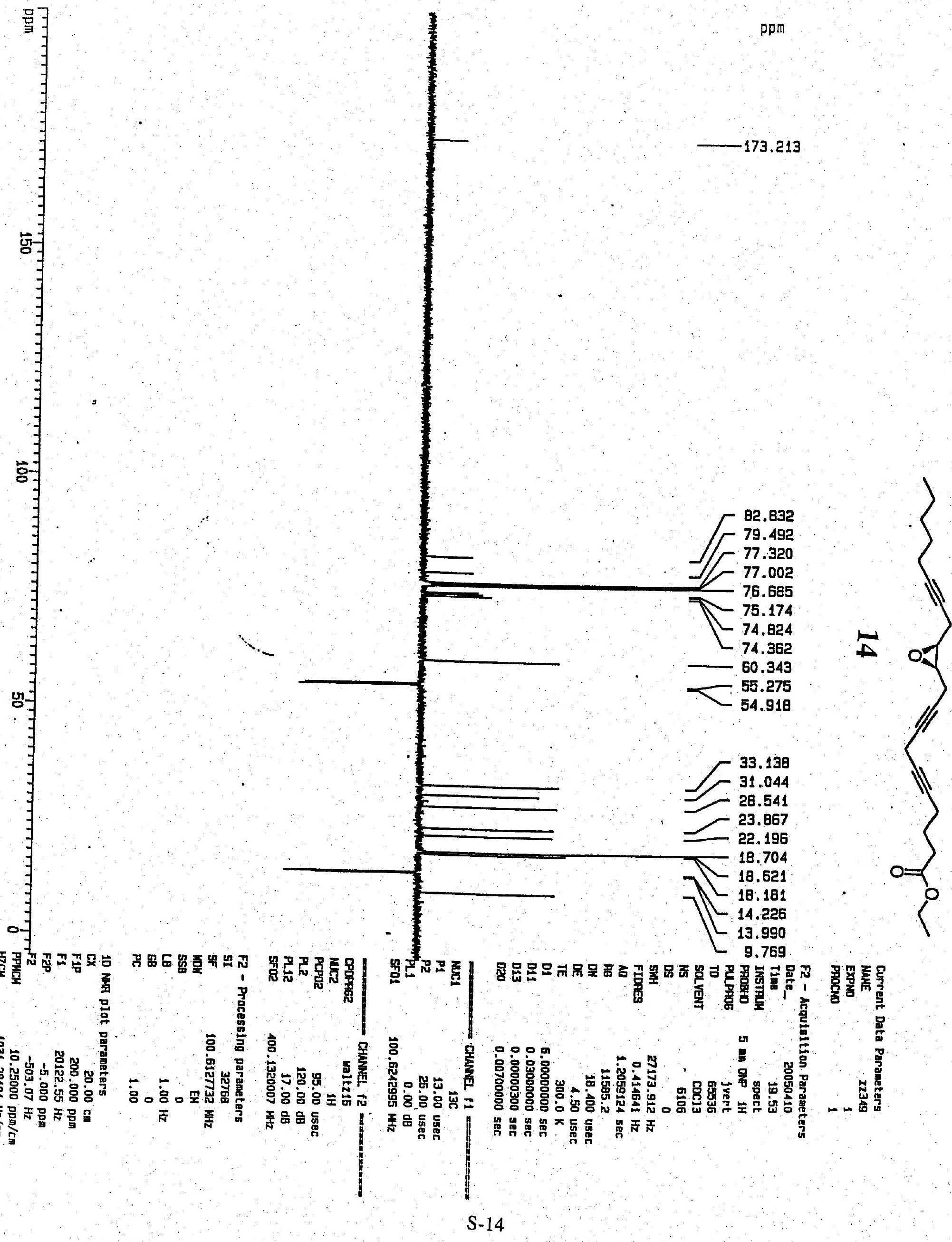



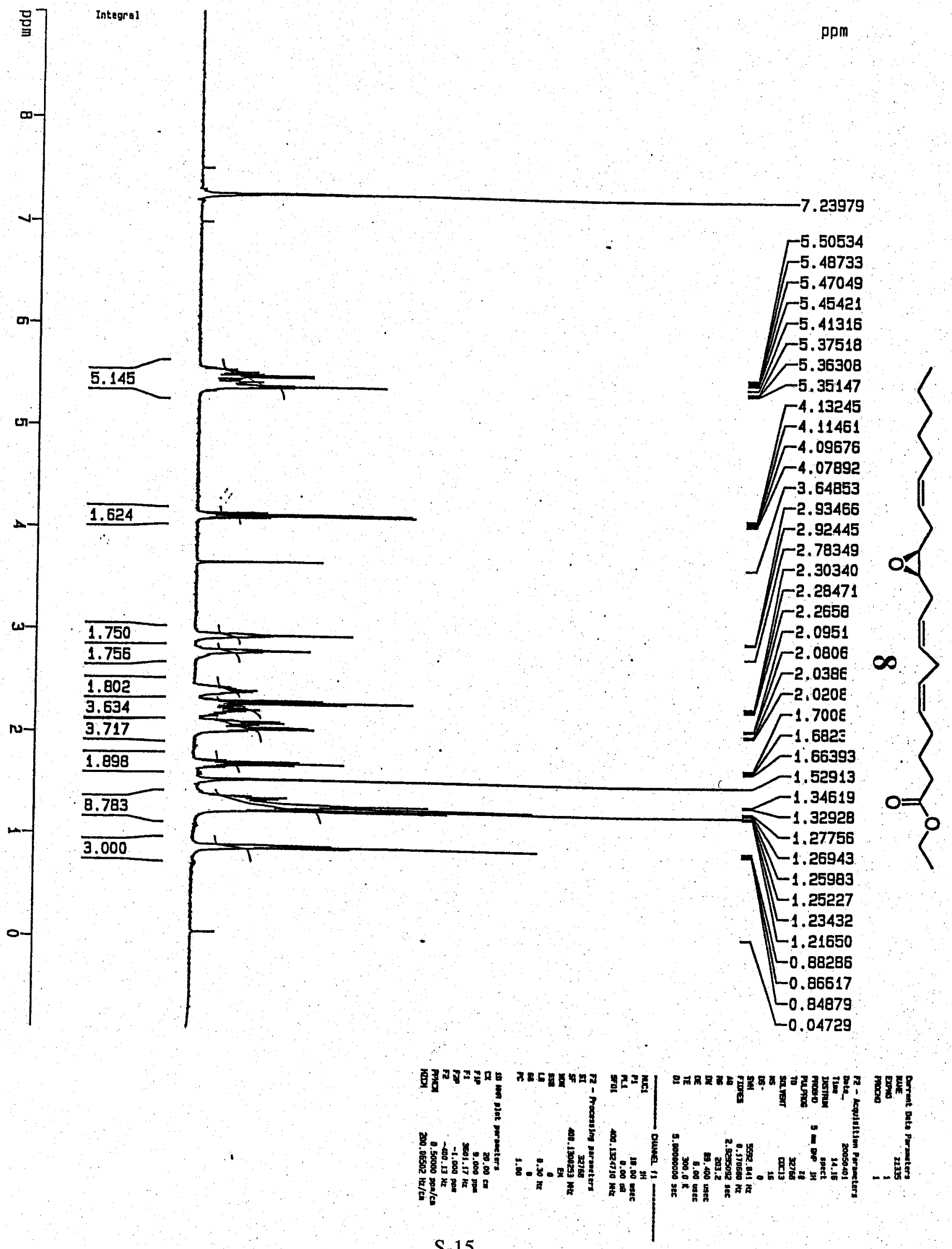


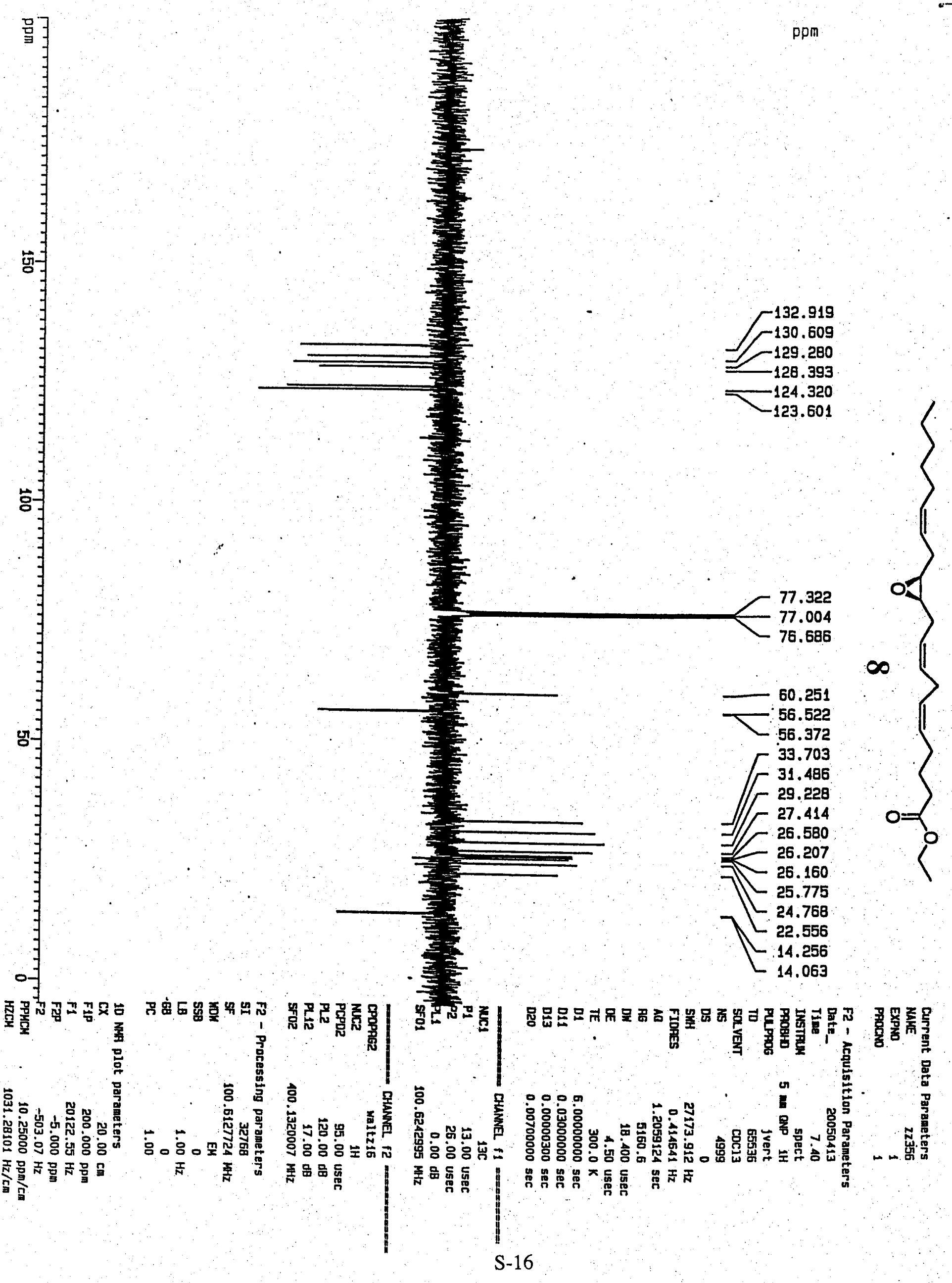



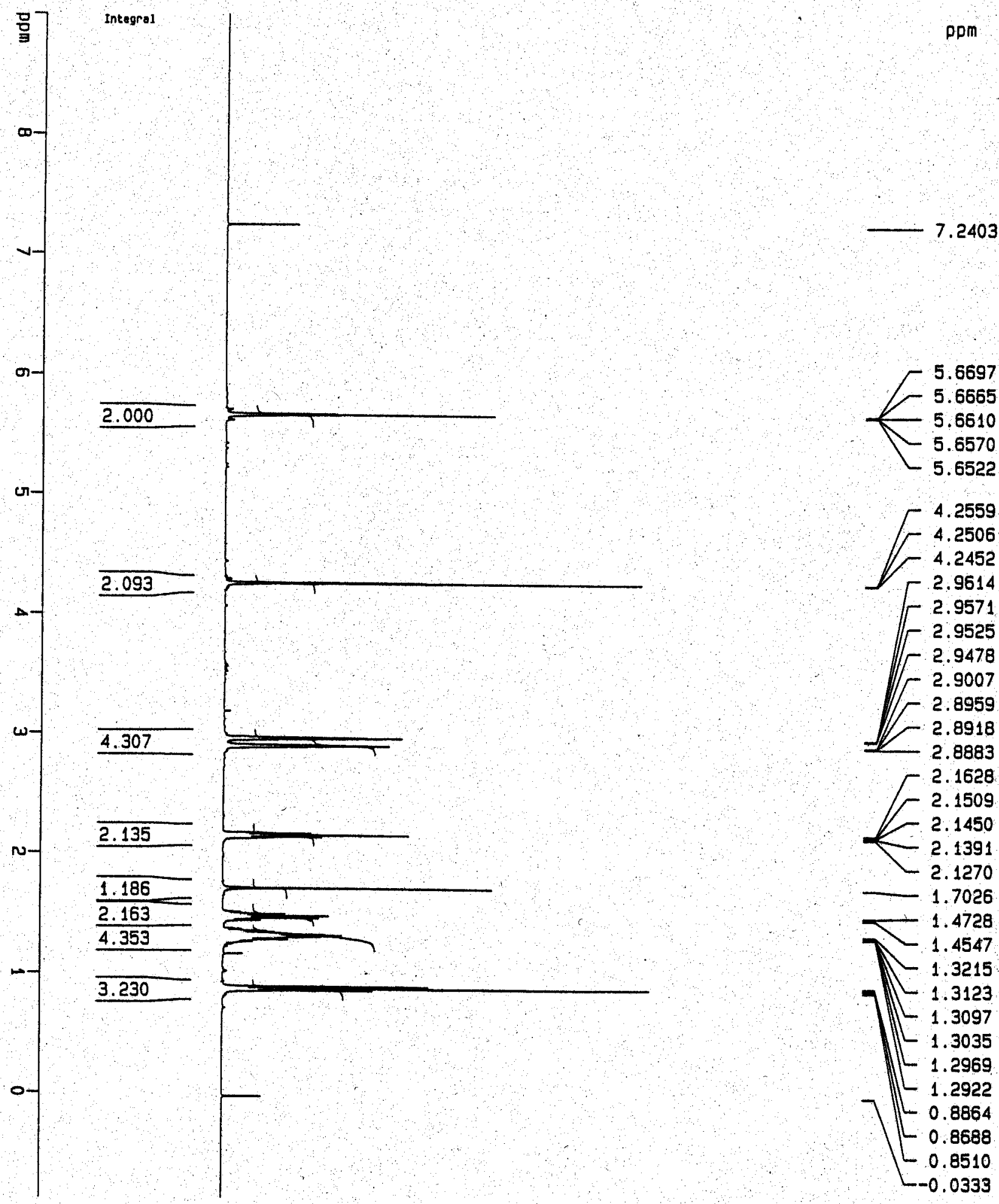

[ 2,9614

$-2.9571$

$-2.9525$

- 2.9478

r 2.9007

- 2.8959

2.8918

2.8883

- 2.1628

$-2.1509$

2. 1450

2.1391

2.1270

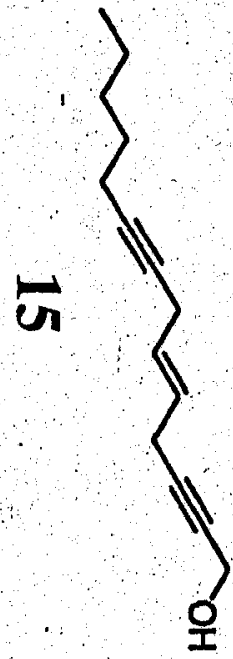

1.7026

1.4728

1.4547

$-1.3215$

] -1.3123

$-1.3097$

$-1.3035$

L 1.2969

$-1.2922$

$-0.8864$

$-0.8688$

$-0.8510$

$-0.0333$

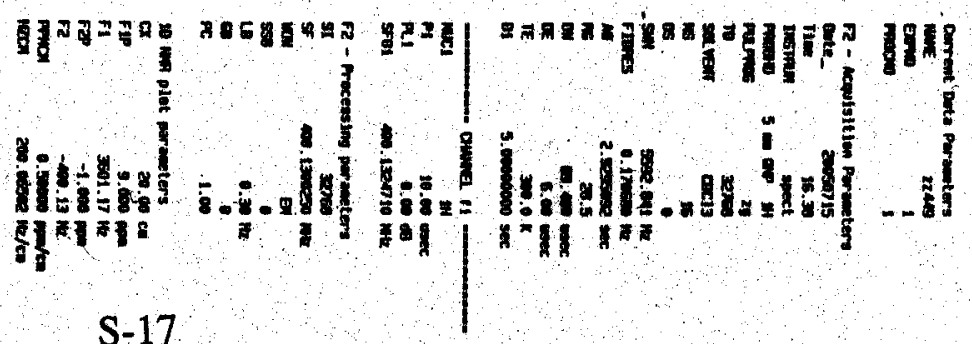




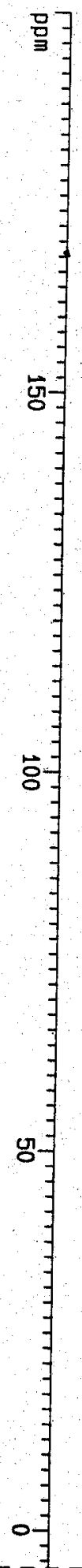

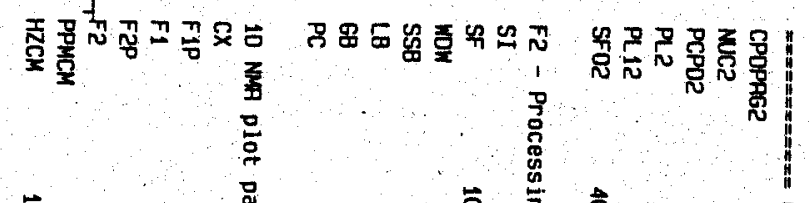

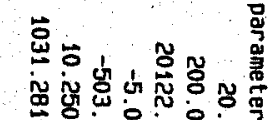

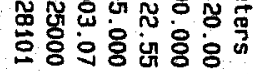

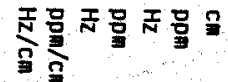

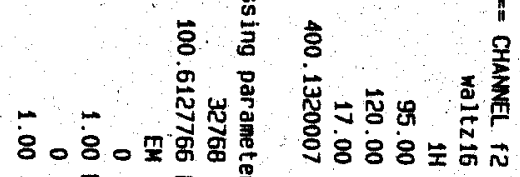

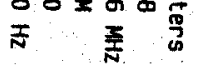

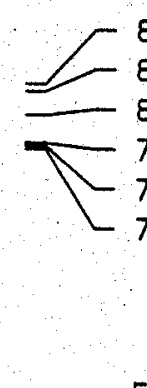

현

$888 \%$

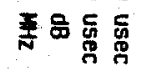

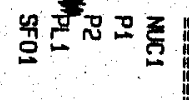

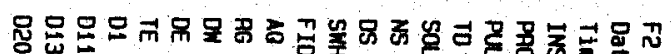

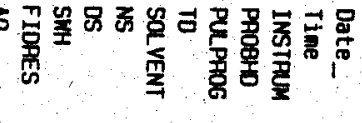

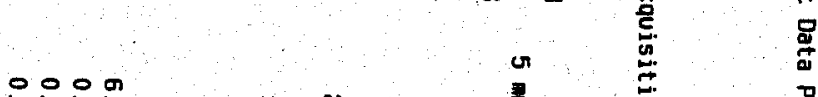

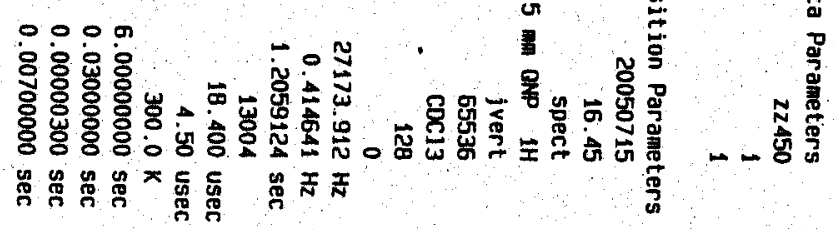

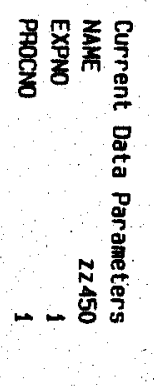



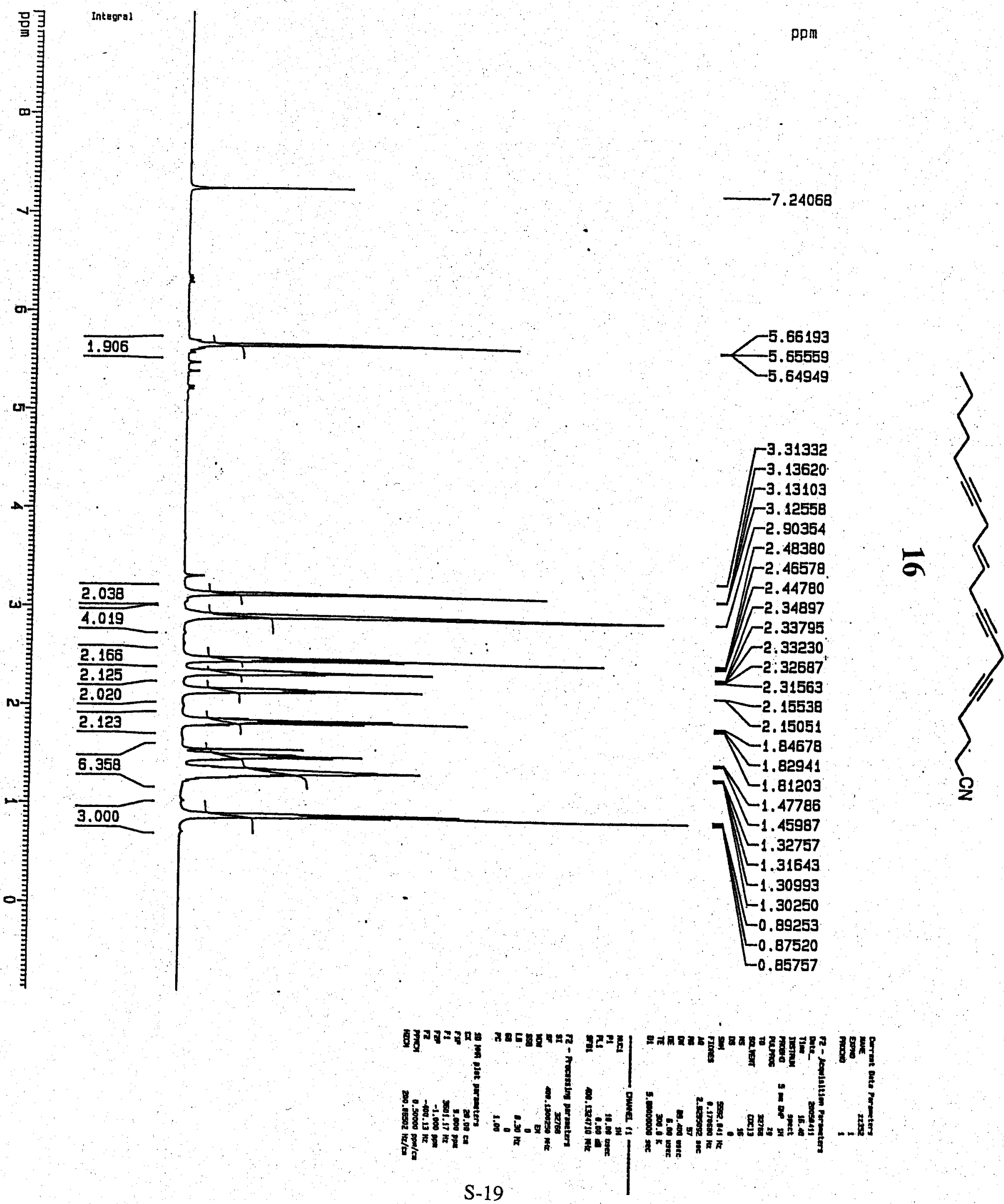


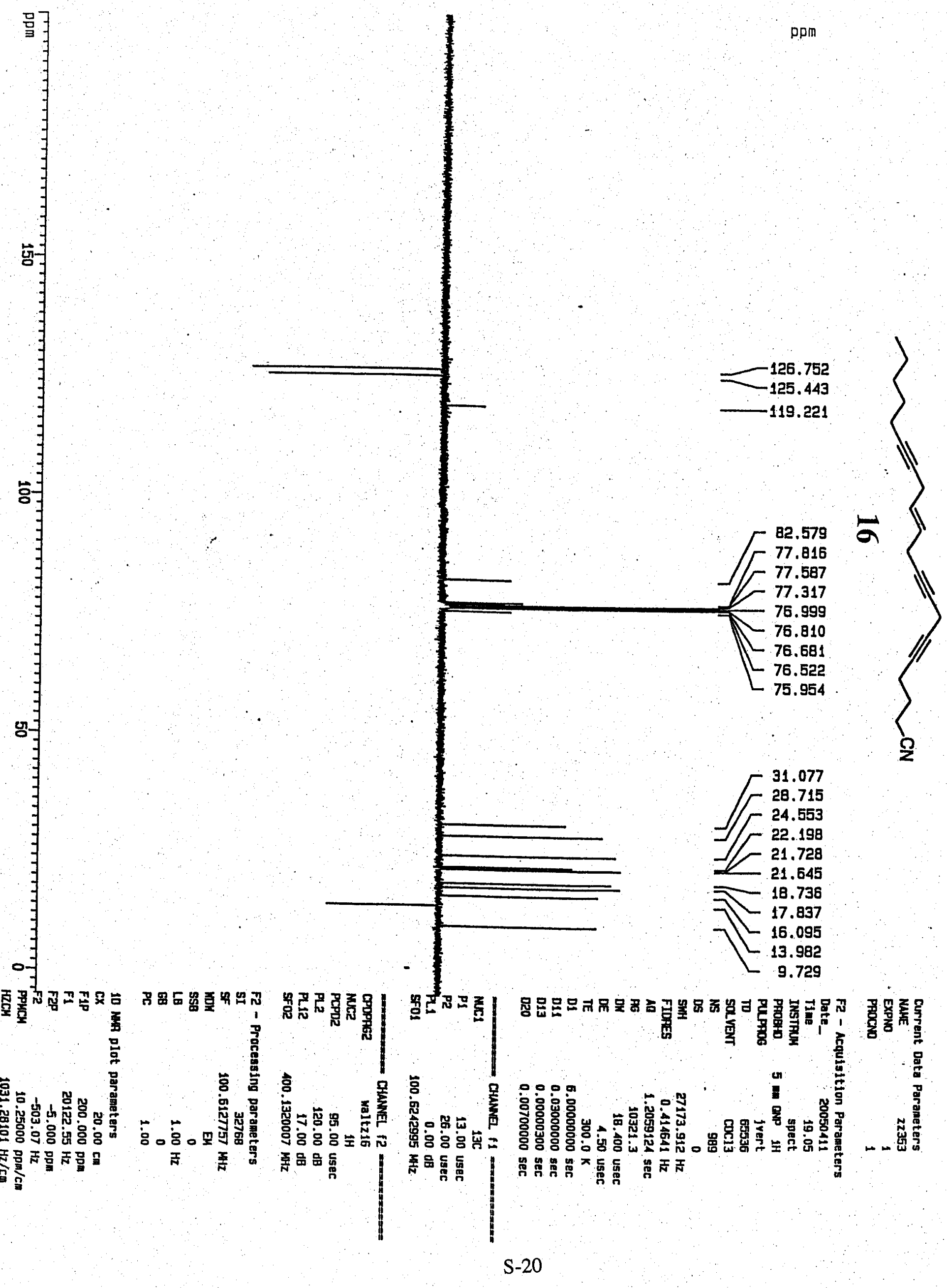



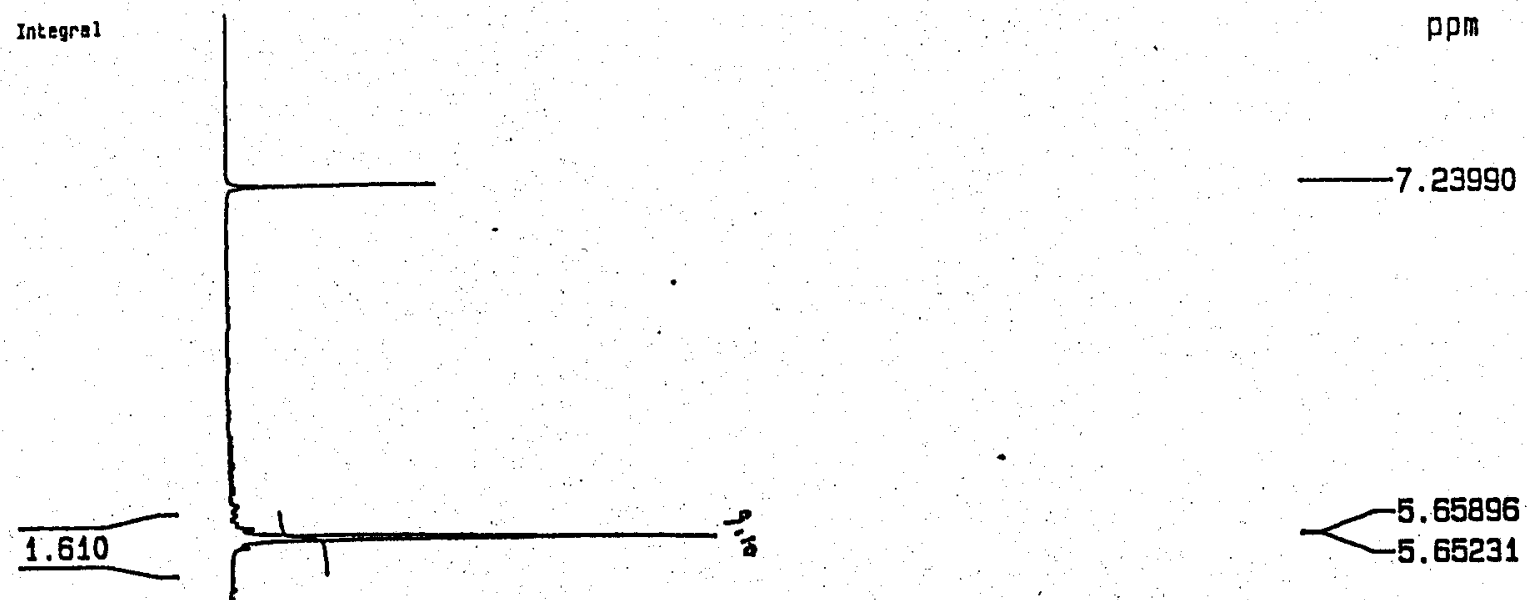

$-5.65231$

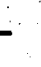

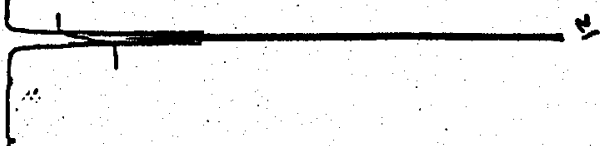

$-3.12235$

$-3.11686$

$-2.89657$

$-2.40473$

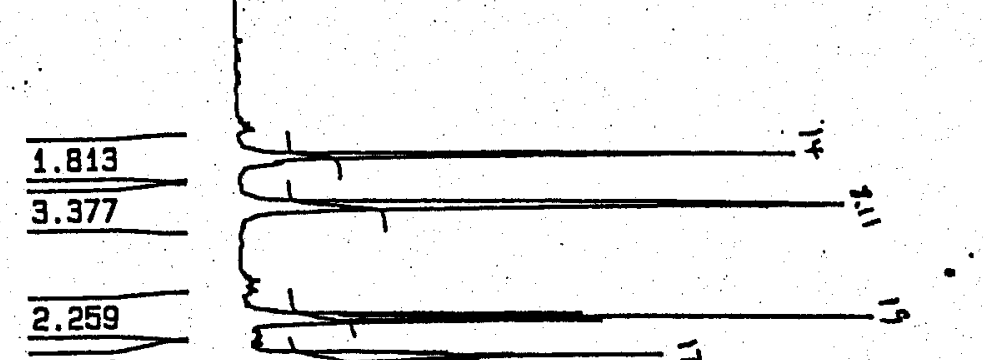

$-2.38599$

$-2.36728$

3.936

N

\begin{tabular}{l}
$\frac{3.017}{1.996}$ \\
\hline .140 \\
\hline .000
\end{tabular}

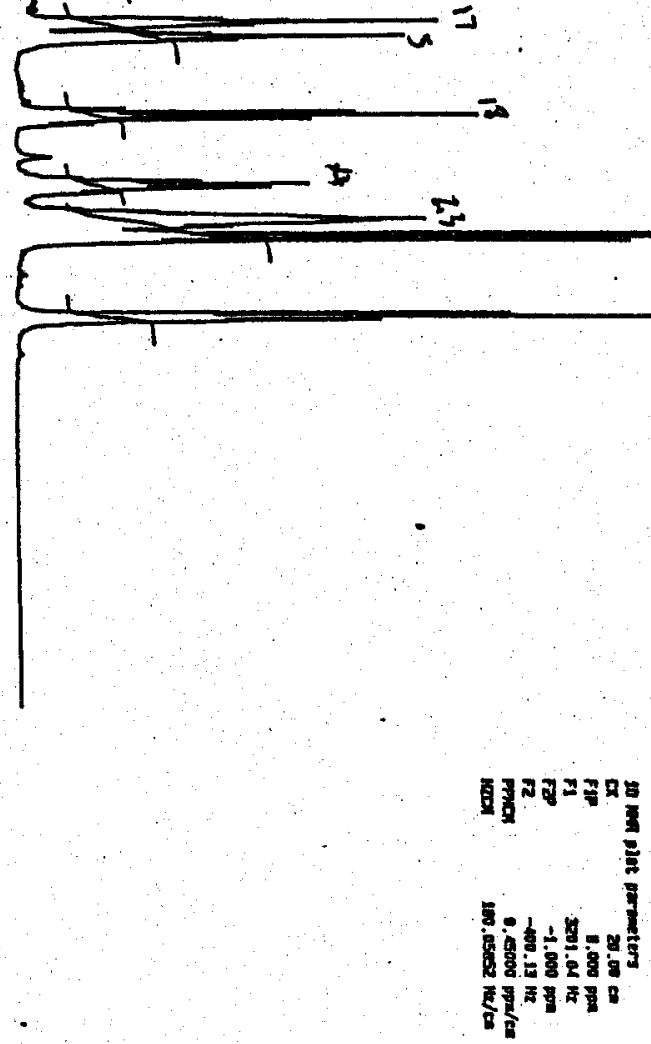

$\infty$

$-2.21567$

$-2.21010$

-2.20462

$-2.14400$

$-2.13893$

$-1.80260$

$-1.78449$

1.76647

$-1.47190$

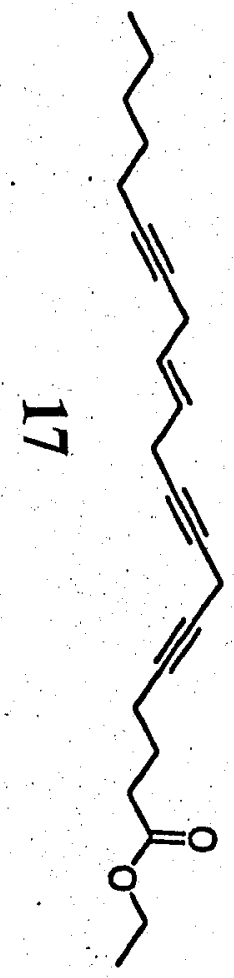




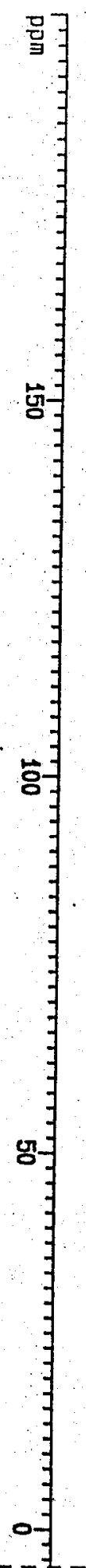

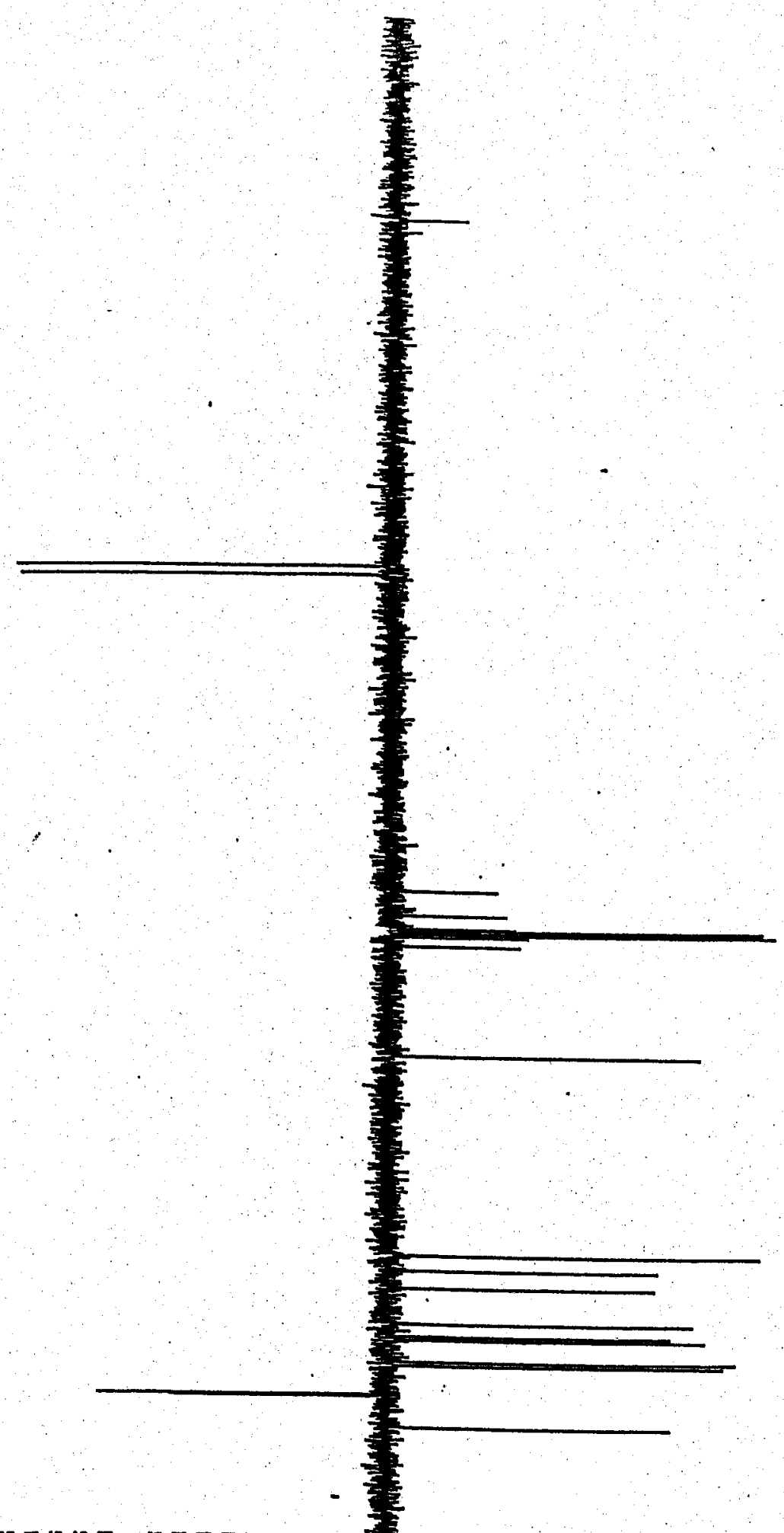

$-173.218$
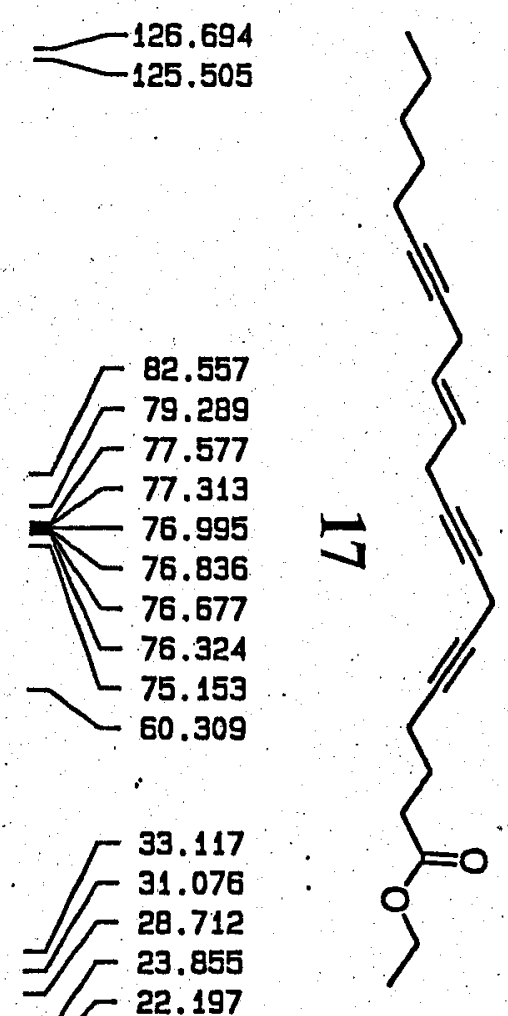

总

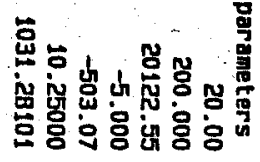

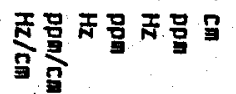

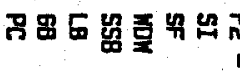

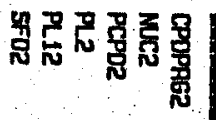

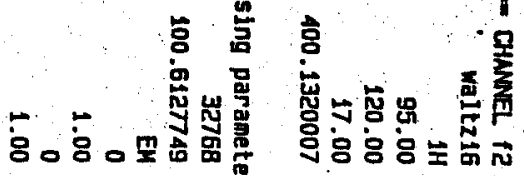
柔畐畐点

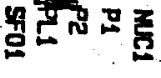

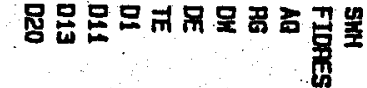

屌

茖

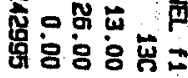

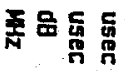

22.197

21.709

21.668

18.735

18. 175

14.201

13.975

9.738 

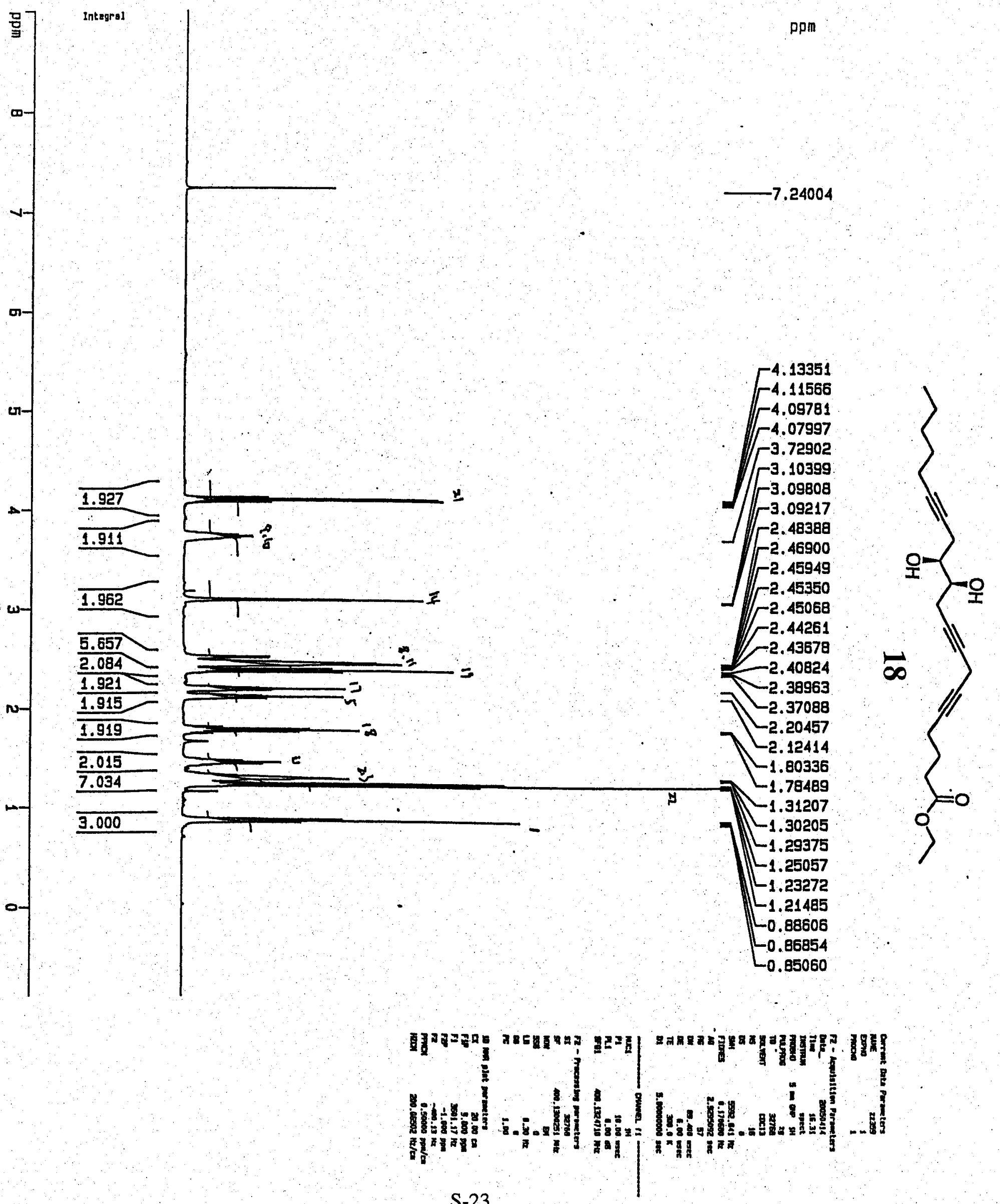


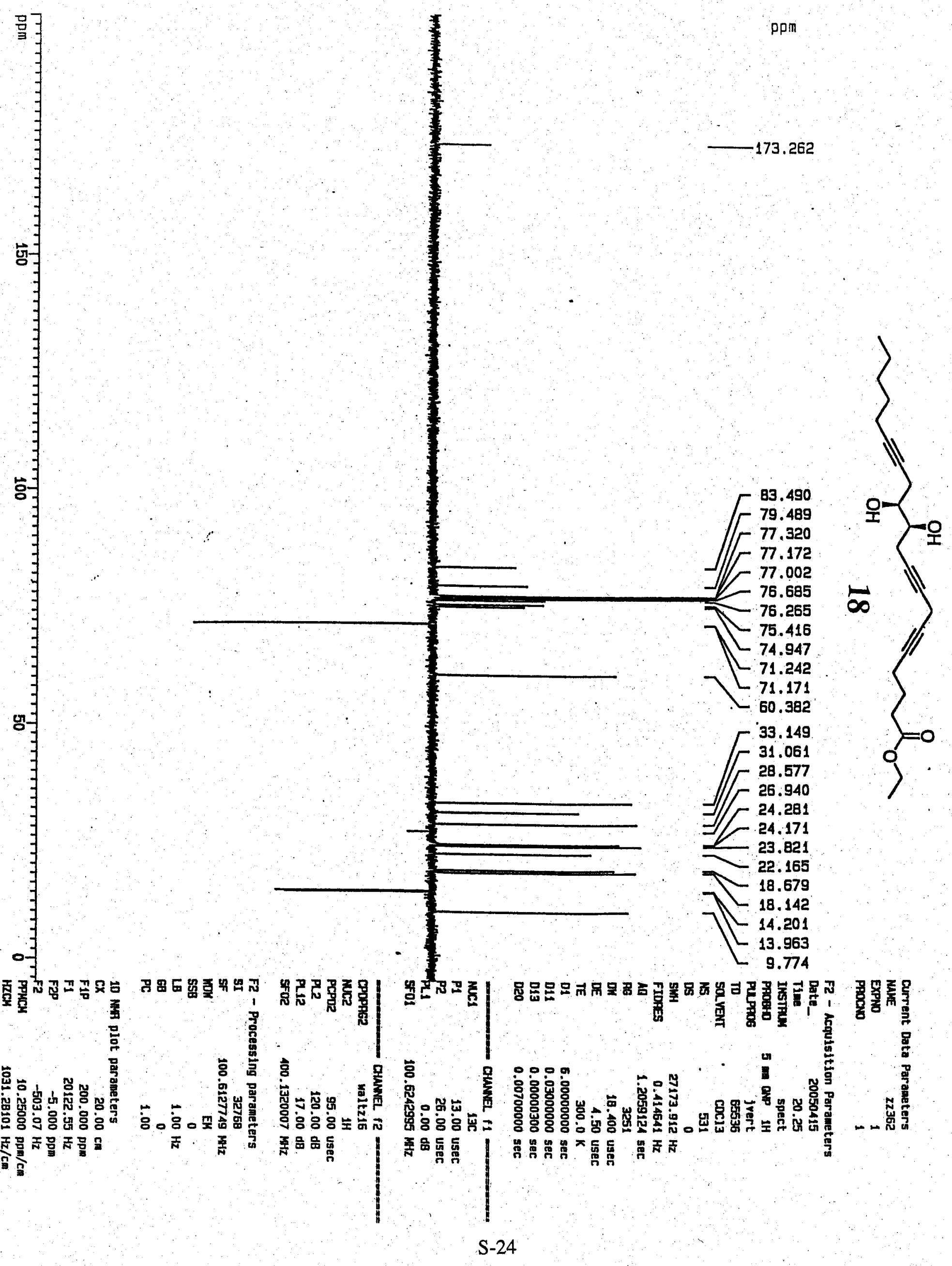



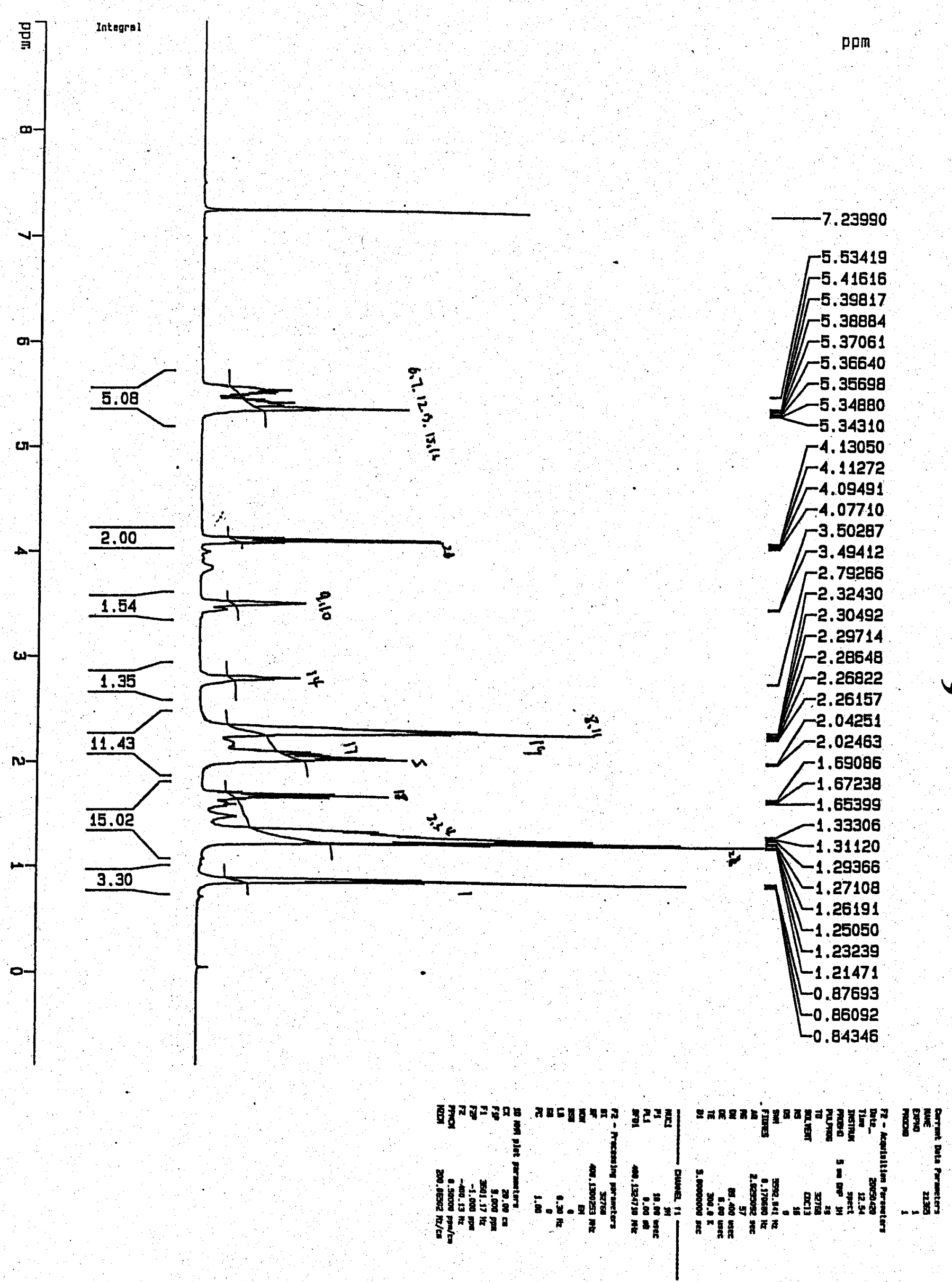


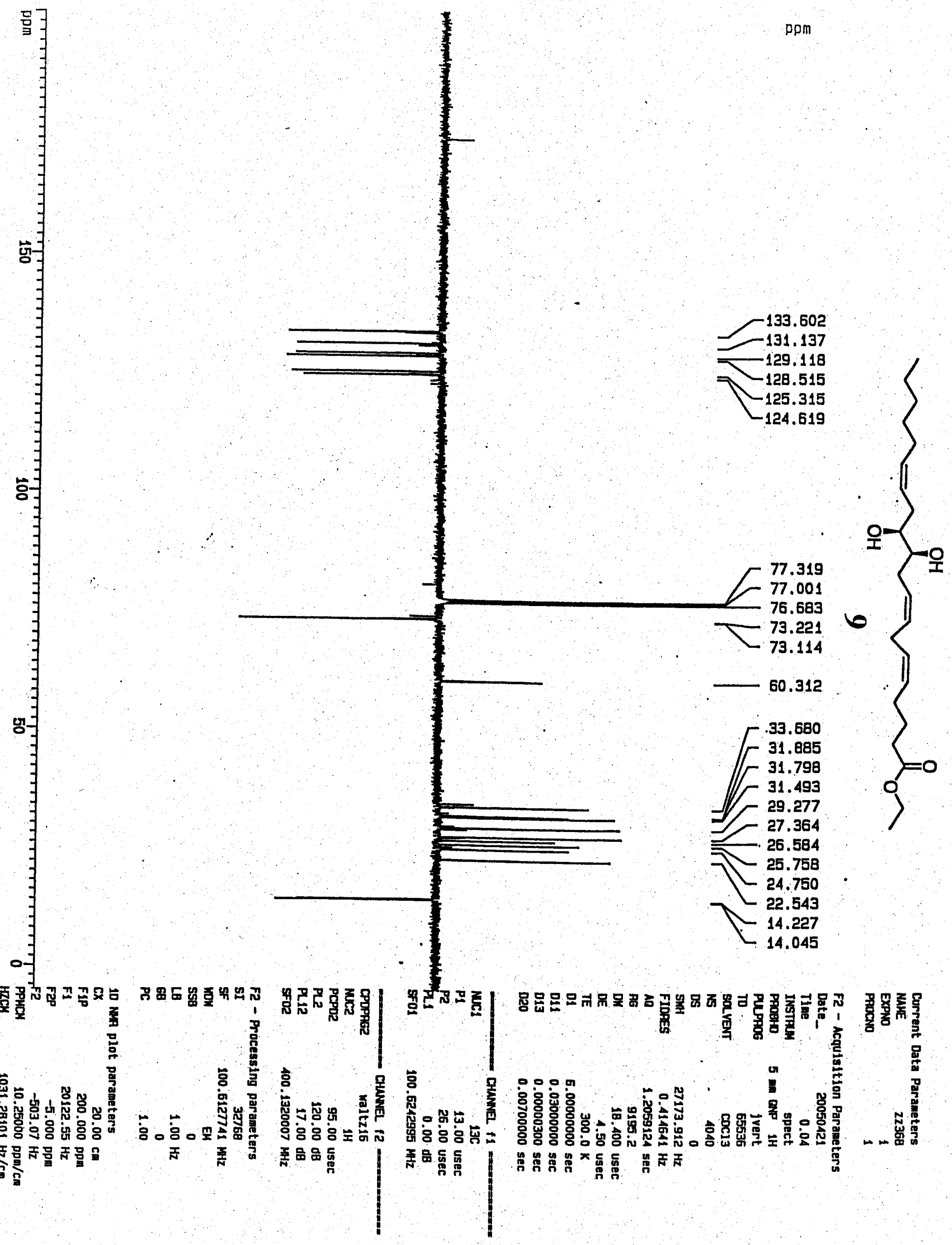



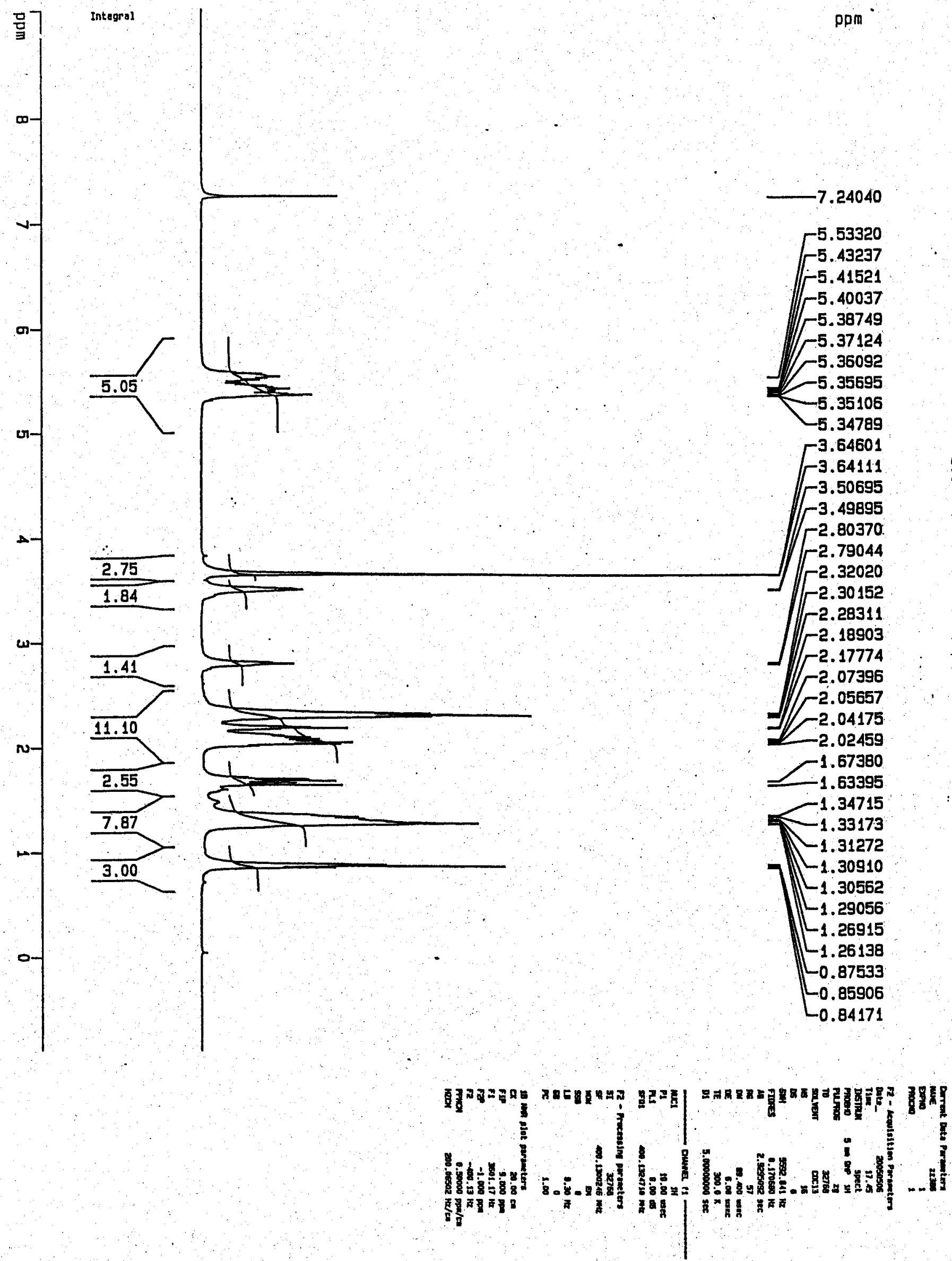


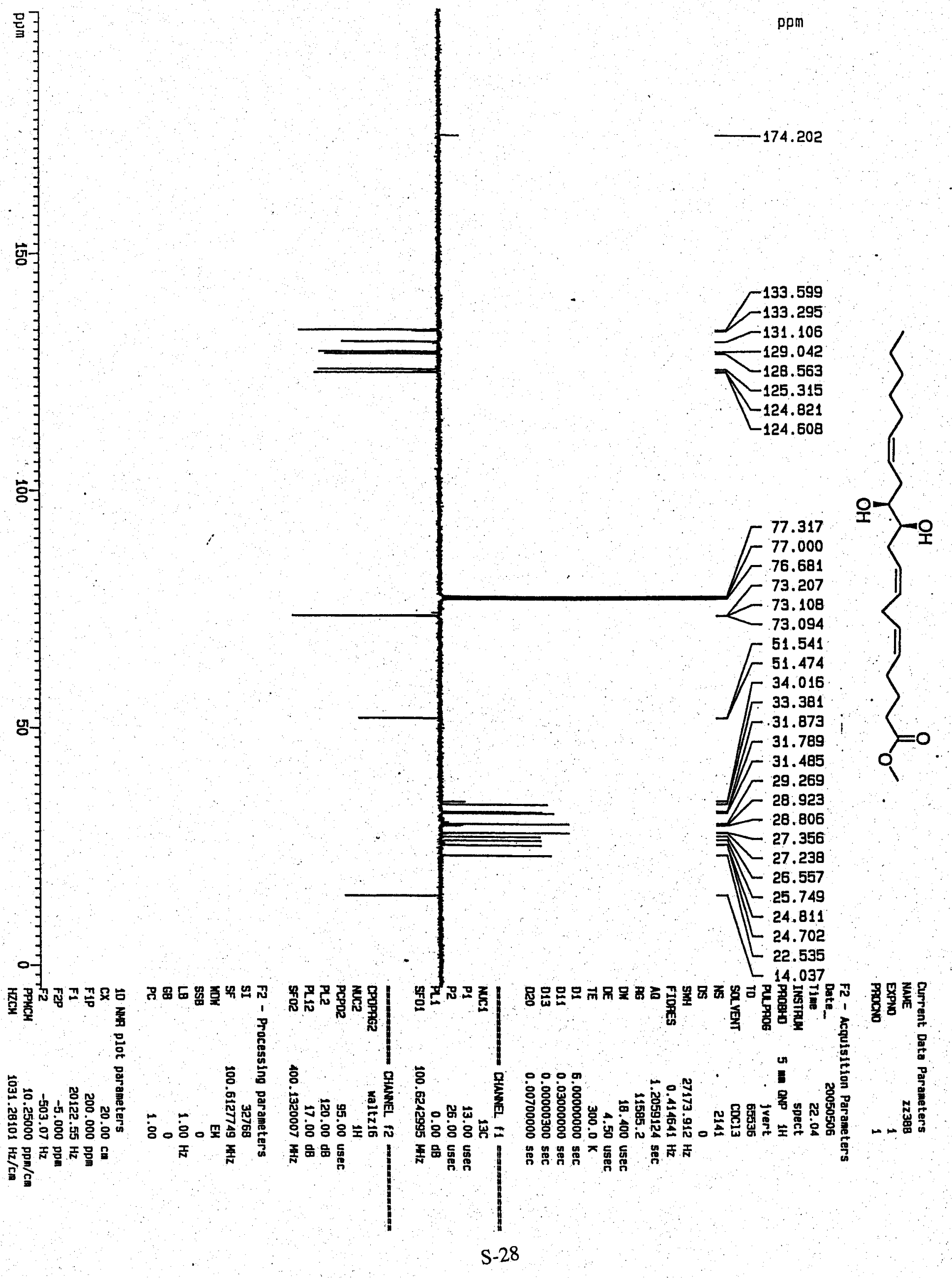

